\title{
Memetic Algorithm with Route Decomposing for Periodic Capacitated Arc Routing Problem
}

\author{
Yuzhou Zhang ${ }^{\mathrm{a}}$, Yi Mei ${ }^{\mathrm{b}, *}$, Ke Tang ${ }^{\mathrm{c}}$, Keqin Jiang ${ }^{\mathrm{a}}$ \\ ${ }^{a}$ School of Computer and Information, Anqing Normal University, Anqing 246133, China \\ ${ }^{b}$ School of Engineering and Computer Science, Victoria University of Wellington, Kelburn 6012, New Zealand \\ ${ }^{c}$ USTC-Birmingham Joint Research Institute in Intelligent Computation and Its Applications (UBRI), School of Computer Science \\ and Technology, University of Science and Technology of China, Hefei 230027, China
}

\begin{abstract}
In this paper, the Periodic Capacitated Arc Routing Problem (PCARP) is investigated. PCARP is an extension of the well-known CARP from a single period to a multi-period horizon. In PCARP, two objectives are to be minimized. One is the number of required vehicles $(n v)$, and the other is the total cost $(t c)$. Due to the multi-period nature, given the same graph or road network, PCARP can have a much larger solution space than the single-period CARP counterpart. Furthermore, PCARP consists of an additional allocation sub-problem (of the days to serve the arcs), which is interdependent with the routing sub-problem. Although some attempts have been made for solving PCARP, more investigations are yet to be done to further improve their performance especially on large-scale problem instances. It has been shown that optimizing $n v$ and $t c$ separately (hierarchically) is a good way of dealing with the two objectives. In this paper, we further improve this strategy and propose a new Route Decomposition (RD) operator thereby. Then, the RD operator is integrated into a Memetic Algorithm (MA) framework for PCARP, in which novel crossover and local search operators are designed accordingly. In addition, to improve the search efficiency, a hybridized initialization is employed to generate an initial population consisting of both heuristic and random individuals. The MA with RD (MARD) was evaluated and compared with the state-of-the-art approaches on two benchmark sets of PCARP instances and a large data set which is based on a real-world road network. The experimental results suggest that MARD outperforms the compared state-of-the-art algorithms, and improves most of the best-known solutions. The advantage of MARD becomes more obvious when the problem size increases. Thus, MARD is particularly effective in solving large-scale PCARP instances. Moreover, the efficacy of the proposed RD operator in MARD has been empirically verified.
\end{abstract}

Keywords: Periodic capacitated arc routing problem, combinatorial optimization, metaheuristics, memetic algorithms

\section{Introduction}

The Capacitated Arc Routing Problem (CARP), presented by Golden and Wong [1, has many practical applications such as mail delivery, street sweep, waste collection, multicommodity network design and hub location [2], 3]. It is a classic combinatorial optimization problem, which deals with a connected undirected graph $G(V, E)$ with

\footnotetext{
${ }^{*}$ Corresponding author

Email address: yi.mei@ecs.vuw.ac.nz (Yi Mei)

Preprint submitted to Journal of LATEX Templates
}

September 8, 2016 
fleet of identical vehicles based at a special vertex called the depot vertex. The objective of CARP is to search for a set of routes with minimal total cost, so that each route begins and ends at the depot vertex, each task is served exactly once, and the total demand of the tasks served by each route must not exceed the vehicle capacity.

CARP is NP-hard [1, and exact methods for CARP such as branch-and-bound [4 can only deal with small and medium sized instances [5]. Heuristics and meta-heuristics are promising methods for tackling the real-world scenarios, in which the problem size is usually large. A number of constructive heuristics have been proposed for CARP, such as augment-merge [1], path-scanning [6], Ulusoy's tour splitting method [7, construct-and-strike [8], and augment-insert [9]. However, these heuristics still cannot obtain satisfying results. In contrast, by searching in the solution space, meta-heuristics manage to obtain much better results with more computational effort. These include tabu search [10, 11], 12], variable neighborhood search [13, 14], guided local search [15], 16], memetic algorithm (MA) [5], [17, [18, [19], 20], 21], bi-objective genetic algorithm [22, cooperative co-evolution [23, simulated annealing 24, and ant colony optimization [25, to name a few. A survey on the CARP can be found in 26.

Although numerous researches on the basic single-period CARP model have been carried out, it is not enough for real-world applications such as waste collection. In waste collection, there are various factors contributing to the amount of refuse production on a street, such as the region, population density, the habit, etc. As a result, the refuse accumulates at different rates on different streets, and the frequency of service varies from street to street. For example, the rural areas produce refuse more slowly than the urban and residential areas. Therefore, it would be reasonable to collect the waste for different types of areas with different frequencies, e.g. daily for the urban and residential areas but every two days for the rural areas. This leads to the Periodic CARP (PCARP) variant, which makes a schedule over a multi-period horizon.

In PCARP, assuming that there are $n p$ periods in the horizon, the number of services for a task over the entire horizon is called its service frequency. The basic CARP constraints (e.g. each route starts and ends at the depot vertex, and the total demand of the tasks in each route cannot exceed vehicle's capacity) must be satisfied. For each task, the service days must belong to some predefined set of service days, and its demand accumulates from its last service day. PCARP is to design an optimal plan that assigns the services of each task to appropriate periods over the horizon, and schedules a set of routes in each period.

Obviously, PCARP reduces to CARP if there is only one period. Since CARP is NP-hard 1], PCARP is NP-hard as well. Moreover, compared to CARP, PCARP has an additional objective besides the original objective (i.e., the , which is to minimize the number of required vehicles $n v$, since the waste management companies attempt to minimize the investment cost (depending on the fleet size) 27]. The additional objective $n v$ affects both the service day allocation (to have balanced number of routes over the horizon) and routing in each period, and imposes extra challenges in PCARP.

As a relatively new extended version of CARP, PCARP attracted much less attention than the traditional model, despite of the fact that it is closer to reality. To the best of our knowledge, there are only a few publications devoted to PCARP so far. In 2002, Lacomme et al. gave the definition of PCARP firstly [27. Thereafter, Chu et al. presented lower bounds in 2003 [28, a linear programming model in 2004 [29] and several constructive heuristics in 2005 [30] for PCARP. In 2005, Lacomme et al. proposed A Memetic Algorithm (MA) (referred to as LMA hereafter), in which a problem-specific crossover operator (i.e., the Periodic Linear Order Crossover, PLOX) was designed [31. Three 45 heuristics were presented and embedded in a Scatter Search (SS) by Chu et al. in 2006, and a greedy constructive heuristic (i.e., the Best Insertion Heuristic, BIH) was used to improve the performance of the initial population [32]. In 2009, a new ant colony system (ACS) algorithm with a heuristic for inserting the tasks into the solutions was 
proposed by Kansou and Yassine [33. However, as an important objective, $n v$ can hardly be improved by above methods, since it is insensitive to the traditional small-step-size search operators. In 2011, Mei et al. developed a -Merging (RM) operator for improving $n v$ particularly. By including the RM operator before the local search process, the MA with RM (MARM) first improves a solution in $n v$, and in $t c$ by the subsequent local search [34. In 2015, Laporte et al. investigated the CARP extensions including the PCARP [35]. Other works include [36], [37] and 38 .

On the other hand, there have been a number of studies proposed on VRP (e.g. [39, 40], 41], 42], 43]) and However, these works are not directly applicable to PCARP due to following reasons: (a) the objective $n v$ was ignored in PVRP, (b) the instance size will becomes much larger, e.g. a CARP with $n$ tasks can be converted to a VRP with $2 n+1$ vertices [48], and (c) the task demand is constant in VRP, but depends on the gap between two successive services in PCARP.

In literature, it is usually assumed that $n v$ is more important than $t c$ in PCARP. In other words, in PCARP, $n v$ is considered to be the primary objective, and $t c$ is considered to be the secondary objective. Under such a consideration, one can combine these two objectives into a single objective by the weighted sum approach and assign a sufficiently large weight to $n v$ to ensure its priority. A previously designed problem-specific operator called the Route Merging (RM) operator has been demonstrated to be promising by improving $n v$ and $t c$ separately during the optimization. However, the RM operator cannot handle the tradeoff between the objectives well enough. In this paper, to overcome this drawback, we propose a new Route Decomposition (RD) operator. The RD operator improves the objective $n v$ by decomposing routes and the objective $t c$ by moving the tasks to appropriate positions heuristically. In contrast to the $\mathrm{RM}$ operator, which considers only $n v$, the proposed $\mathrm{RD}$ operator takes both $n v$ and $t c$ into account while still keeping the priority of $n v$. This way, the RD operator optimizes both $t c$ and $n v$ simultaneously. More specially, to reduce $n v, \mathrm{RD}$ selects one route from the period with the most routes to decompose. Then, to reduce $t c$, it moves the tasks on the selected route to new positions to minimize the additional tc value. Based on the RD operator, a novel MA with RD (MARD) is proposed. In addition, to improve search efficiency, two constructive heuristics (i.e., BIH and path-scanning) are employed to generate the initial population. The experimental studies demonstrate that MARD can obtain better solutions than the state-of-the-art algorithms on the tested benchmark sets and has a significantly better performance in terms of both $n v$ and $t c$ on large-scale instances.

The remainder of this paper is organized as follows. Section 2 gives the notations and problem model. In Section 3 , the relationship between the two objectives is investigated and the RD operator is proposed. Section 4 introduces our new approach MARD in detail, followed by experimental studies in Section 5. Finally, the conclusions are presented in Section 6.

\section{Problem Model of PCARP}

In PCARP, consider an undirected connected graph $G(V, E)$ and a horizon $H$ of $n p$ periods, where $V$ and $E$ are the vertex (e.g. crossroad) and edge (e.g. street) sets. There are $m$ identical vehicles with limited capacity $Q$ at the depot vertex $v_{0} \in V$. Each edge $e \in E$ has four non-negative attributes: the demand vector $\vec{d}(e)=\left(d_{1}(e), d_{2}(e), \ldots, d_{n p}(e)\right)$, the service frequency $\eta(e)$, the serving cost $s c(e)$ and the deadheading cost $d c(e)$. In $\vec{d}(e), d_{p}(e)$ indicates the demand of $e$ in period $p$. The service frequency $\eta(e)$ is the number of required services during horizon. The serving $\operatorname{cost} s c(e)$ means the cost induced by serving $e$, and the deadheading cost $d c(e)$ is the cost for traversing $e$ without service. Each task $t$ in the task set $E_{R} \subseteq E$ must be served in at least one period. That is, $\eta(t) \geq 1$, and $s c(t)>0$. Each 
task $t$ has an allowed period combination set $\mathcal{A P C}(t)$, which consists of a number of period combinations. A period combination $P C(t)=\left\{p_{1}(t), \ldots, p_{\eta(t)}(t)\right\} \in \mathcal{A P C}(t)$ is a set of $\eta(t)$ periods, indicating the periods that $t$ is served. Each task $t$ is assigned two positive integer IDs $t_{1}$ and $t_{2}$, one for each direction. For ID $t_{i}$, let $i n v\left(t_{i}\right)$ stand for its reverse direction and they are equivalent in the service frequency, serving cost, deadheading cost and demand, but it is important to note that only one of them can be served in a period. In addition, $h e a d\left(t_{i}\right)$ and $\operatorname{tail}\left(t_{i}\right)$ denote the head and tail vertices respectively.

For PCARP, a route $R_{p k}$, which is traversed by vehicle $k$ in period $p$, can be represented as a sequence of tasks. That is,

$$
R_{p k}=\left(0, t_{p k 1}, t_{p k 2}, \ldots, t_{p k l_{p k}}, 0\right)
$$

where $l_{p k}$ is the number of tasks in $R_{p k}$. A dummy loop $\left(v_{0}, v_{0}\right)$ is created to ensure the route to start and end at the depot and assigned an ID 0, i.e. $\operatorname{tail}(0)=h e a d(0)=v_{0}$. The number of routes in period $p$ is denoted as $n r_{p}$. If the task $t$ appears in the sequence of $R_{p k}\left(k=1, \ldots, n r_{p}\right)$, then $p \in P C(t)$. Otherwise, $p \notin P C(t)$. Then, a solution $S$ to PCARP can be represented as a route set, i.e.,

$$
S=\left\{R_{p k} \mid p=1,2, \ldots, n p ; k=1,2, \ldots, n r_{p}\right\}
$$

In PCARP, we assume that the demand of each task accumulates over time in a periodic manner. That is, the remaining demand from the previous period (e.g. week) will be inherited to the next one. Therefore, given a period combination $P C(t)$ of the task $t$, the steady-state accumulated demand $a d(t, p)$ of task $t$ in period $p$ can be computed as follows:

Step 1. Initialize $i=p, \operatorname{ad}(t, p)=d_{i}(t)$;

Step 2. $i \leftarrow i-1$. If $i=0$, set $i=n p$;

Step 3. If $p_{i}(t) \notin P C(t), a d(t, p) \leftarrow a d(t, p)+d_{i}(t)$, and go to Step 2;

Step 4. Return $a d(t, p)$.

The total cost and load of each route $R_{p k}$ can be computed as:

$$
\begin{aligned}
& \operatorname{cost}\left(R_{p k}\right)=\sum_{i=1}^{l_{p k}} s c\left(t_{p k i}\right)+\sum_{i=0}^{l_{p k}} \delta\left(\operatorname{tail}\left(t_{p k i}\right), \operatorname{head}\left(t_{p k(i+1)}\right)\right) \\
& \operatorname{load}\left(R_{p k}\right)=\sum_{i=1}^{l_{p k}} a d\left(t_{p k i}, p\right)
\end{aligned}
$$

where $t_{p k 0}=t_{p k\left(l_{p k}+1\right)}=0$. The function $\delta\left(v_{i}, v_{j}\right)$ is the shortest distance (with minimum deadheading cost) from the vertex $v_{i}$ to $v_{j}$, which can be obtained by Dijkstra's algorithm [49. A vehicle can serve only one route. Hence, for period $p$, the number of vehicles is equal to the number of routes $n r_{p}$.

Then, we can obtain two objectives $t c(S)$ and $n v(S)$ of given solution $S$ to PCARP.

$$
\begin{aligned}
& t c(S)=\sum_{p=1}^{n p} \sum_{k=1}^{n r_{p}} \operatorname{cost}\left(R_{p k}\right) \\
& n v(S)=\max \left\{n r_{p} \mid p=1, \ldots, n p\right\}
\end{aligned}
$$

According to the above definitions, the PCARP model can be described as below:

$$
\min f(S)=\alpha \cdot n v(S)+t c(S)
$$




$$
\begin{aligned}
& \text { s.t. }: \operatorname{count}\left(t_{i}, p\right)+\operatorname{count}\left(\operatorname{inv}\left(t_{i}\right), p\right) \leq 1, t_{i} \in\{\text { IDs of } t\}, t \in E_{R}, p=1,2, \ldots, n p \\
& \sum_{p=1}^{n p}\left(\operatorname{count}\left(t_{i}, p\right)+\operatorname{count}\left(\operatorname{inv}\left(t_{i}\right), p\right)\right)=\eta(t), t_{i} \in\{\text { IDs of } t\}, t \in E_{R} \\
& P C(t) \in \mathcal{A P C}(t), t \in E_{R} \\
& \operatorname{load}\left(R_{p k}\right) \leq Q, p=1,2, \ldots, n p, k=1,2, \ldots, n r_{p}
\end{aligned}
$$

where $\operatorname{count}\left(t_{i}, p\right)$ denotes the times that task $t$ appears in the period $p$ at the direction denoted by its ID $t_{i}$. In PCARP, $n v$ is considered to be more important than $t c$. Therefore, the coefficient $\alpha$ in Eq. (7) is set to a sufficiently large value to ensure the priority of $n v$. Constraint (8) shows that each task $t$ is served no more than once in one period, and its service frequency over the horizon $H$ can be guaranteed by constraint (9). Constraint (10) indicates that the arranged period combination of each task belongs to its allowed period combination set. Constraint (11)

\begin{tabular}{|c|c|}
\hline Symbol & Description \\
\hline$G(V, E)$ & An undirected connected graph \\
\hline$V$ & Vertex set \\
\hline$E$ & Edge set \\
\hline$H$ & Time horizon \\
\hline$n p$ & Number of periods \\
\hline$Q$ & Vehicle capacity \\
\hline$m$ & Number of vehicles available \\
\hline$v_{0}$ & Depot vertex \\
\hline$e$ & An edge \\
\hline$E_{R}$ & Task set \\
\hline$t$ & A task \\
\hline$\vec{d}(e)(\vec{d}(t))$ & Demand vector of edge $e(\operatorname{task} t)$ \\
\hline$\eta(e)(\eta(t))$ & Service frequency of edge $e(\operatorname{task} t)$ \\
\hline$s c(e)(s c(t))$ & Serving cost of edge $e(\operatorname{task} t)$ \\
\hline$d c(e)(d c(t))$ & Deadheading cost of edge $e(\operatorname{task} t)$ \\
\hline$n$ & Number of required edges (tasks) \\
\hline$\gamma$ & Total number of services over $H$ \\
\hline $\mathcal{A P C}(t)$ & Allowed period combination set of task $t$ \\
\hline$P C(t)$ & A period combination of task $t$ represented as a vector \\
\hline$\lambda_{t}$ & Number of the period combinations in $\mathcal{A P C}(t)$ \\
\hline$\delta\left(v_{i}, v_{j}\right)$ & $\begin{array}{c}\text { The shortest distance from vertex } v_{i} \text { to } v_{j} \\
\text { A solution to PCARP }\end{array}$ \\
\hline$R_{p k}$ & A route traversed by vehicle $k$ in period $p$ \\
\hline$l_{p k}$ & Total number of served tasks in $R_{p k}$ \\
\hline $\operatorname{ad}(t, p)$ & Accumulated demand of task $t$ in period $p$ \\
\hline $\operatorname{cost}\left(R_{p k}\right)$ & Cost of route $R_{p k}$ \\
\hline $\operatorname{load}\left(R_{p k}\right)$ & Load of route $R_{p k}$ \\
\hline$n r_{p}$ & Number of routes in period $p$ \\
\hline$n v(S)$ & Number of vehicles used in solution $S$ over horizon $H$ \\
\hline$t c(S)$ & Total cost of solution $S$ \\
\hline$\stackrel{\alpha}{\alpha(S)}$ & $\begin{array}{c}\text { Coefficient of } n v(S) \text { in the objective function } \\
\text { Objective function }\end{array}$ \\
\hline
\end{tabular}
shows that the load (total demand of the served tasks) of each route cannot exceed the capacity $Q$.

For the sake of convenience, all the notations used in this paper are listed in Table 1 for quick reference.

Table 1: Commonly used mathematical notations in this paper

\section{Route Decomposing Operator for PCARP}

Based on the existing methods for CARP, researchers have presented modified algorithms for PCARP, such as LMA and SS. To solve PCARP, these algorithms extended the solution representation and search operators that were designed for CARP. However, the modified approaches can hardly deal with the objective $n v$ of PCARP well, because they mainly focus on minimizing $t c$ alone. For LMA and SS, the improvement of objectives largely depends on the crossover and local search operators. Analysis in 34 has shown that it is hard to get high-quality solutions with smaller $n v$ 's from their parents by crossover and traditional local search with small-step operators that move 


\subsection{Movement of a Task}

The movement of a task $t$ of solution $S$ works is described in Algorithm 2 , First, a new period combination $P C_{\text {new }}(t) \in \mathcal{A P C}(t) \backslash\left\{P C_{\text {curr }}(t)\right\}$ is found by the function selectPeriodCombination() (line 2), which will be 
described in detail next. Then, all the services of $t$ are removed from the periods that do not belong to $P C_{n e w}(t)$. Finally, a service of $t$ is inserted into each period $p \in P C_{n e w}(t) \backslash P C_{\text {curr }}(t)$, in which the service of $t$ is missed (lines 4 26). Here, the service insertion of $t$ are also done in $p_{\text {sel }}$ if $p_{\text {sel }} \in P C_{\text {new }}(t) \cap P C_{\text {curr }}(t)$, because it has been removed from $p_{\text {sel }}$ by Algorithm 1. For inserting $t$ into one period, all the routes and positions are examined (including an empty route) (lines 11,18 ), and the best feasible route $r^{*}$ and position $i^{*}$ that yield the least additional cost is selected to insert the service (lines 20 24. For the sake of convenience, such an insertion strategy is called the best insertion strategy hereafter.

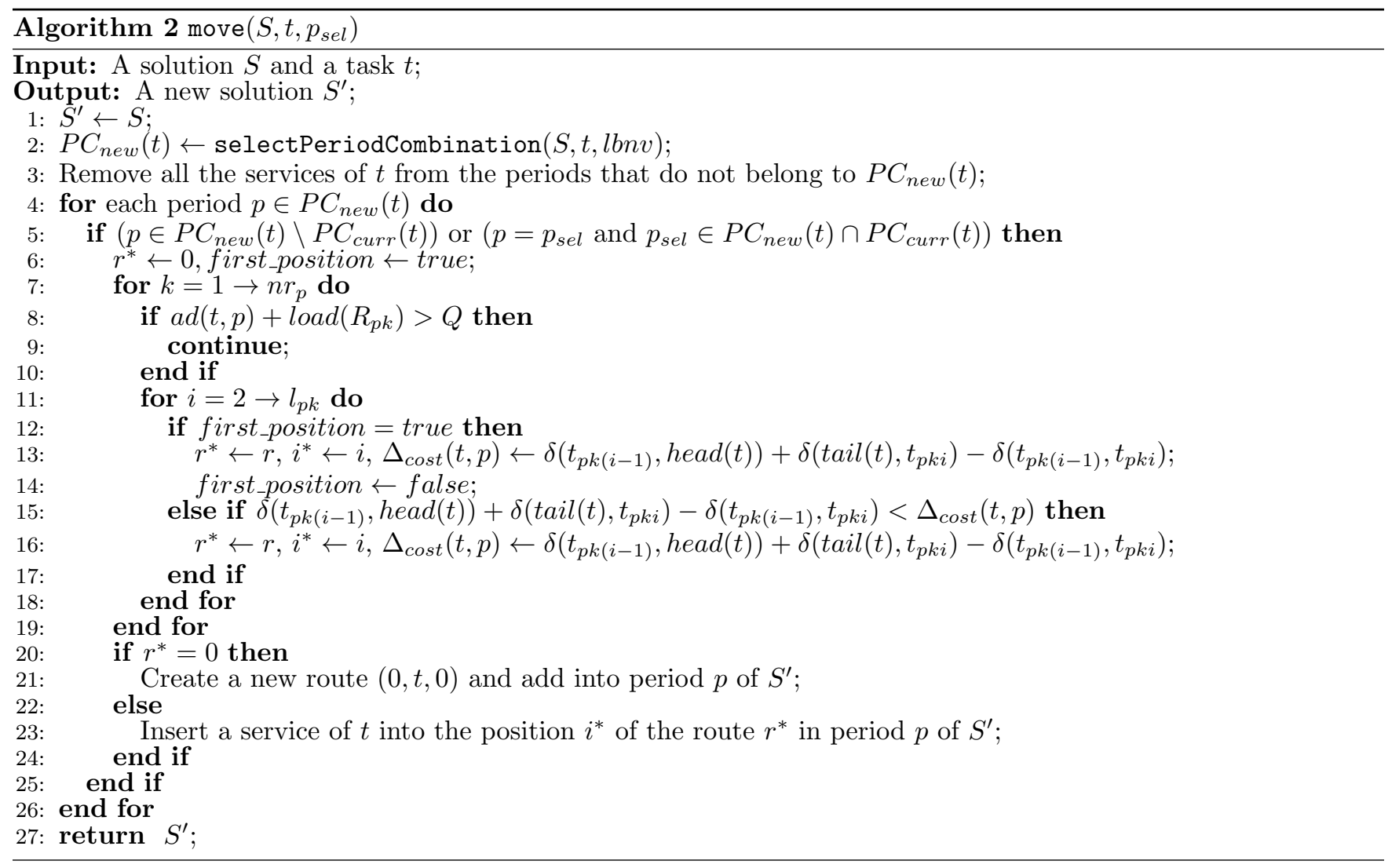

In the function selectPeriodCombination(), a good new period combination should have the following three properties: (1) it overlaps with the current period combination $P C_{c u r r}(t)$ well, $(2)$ the additional cost caused by moving the services from the current period combination to the new one (lines 426 in Algorithm 2 is small, and (3) the maximal number of routes in the periods that the services are to be inserted into, if larger than lbnv, is small. The first property reduces the number of service removal and insertion operations. The second property aims to minimize the cost. The third property tends to reduce the maximal number of vehicles over the horizon.

Based on the above intuition, we define a utility function $U(P C(t))$ for each $P C(t) \in \mathcal{A P C}(t) \backslash\left\{P C_{c u r r}(t)\right\}$ as follows:

$$
U(P C(t))=\frac{C-\Delta_{c o s t}(P C(t))}{\left(\eta(t)-C_{1}(P C(t))\right) \cdot C_{2}(P C(t))}
$$

where $\Delta_{\text {cost }}(P C(t))$ indicates the total additional cost caused by moving all the tasks from $P C_{c u r r}(t)$ to $P C(t)$ (inserted by the best insertion). $C$ is a large constant (3000 in our experiments), which can ensure that $C-$ $\Delta_{\text {cost }}(P C(t)) \geq 0 . \quad C_{1}(P C(t))=\left|P C(t) \cap P C_{\text {curr }}(t)\right|$ is the number of common periods shared by $P C(t)$ and $P C_{\text {curr }}(t) . C_{2}(P C(t))$ is defined as follows:

$$
C_{2}(P C(t))=\max \left\{\max \left\{n r_{p} \mid p \in P C(t) \backslash P C_{c u r r}(t)\right\}-l b n v+1,1\right\}
$$


From Eq. (13), we can see that a $P C(t)$ with a smaller additional cost, more periods shared with $P C_{c u r r}(t)$ or smaller number of routes, tends to have a larger utility function value. Thus, the $P C(t)$ with the largest utility function value is selected as the new period combination. The pseudo code of selectPeriodCombination () is given in Algorithm 3.

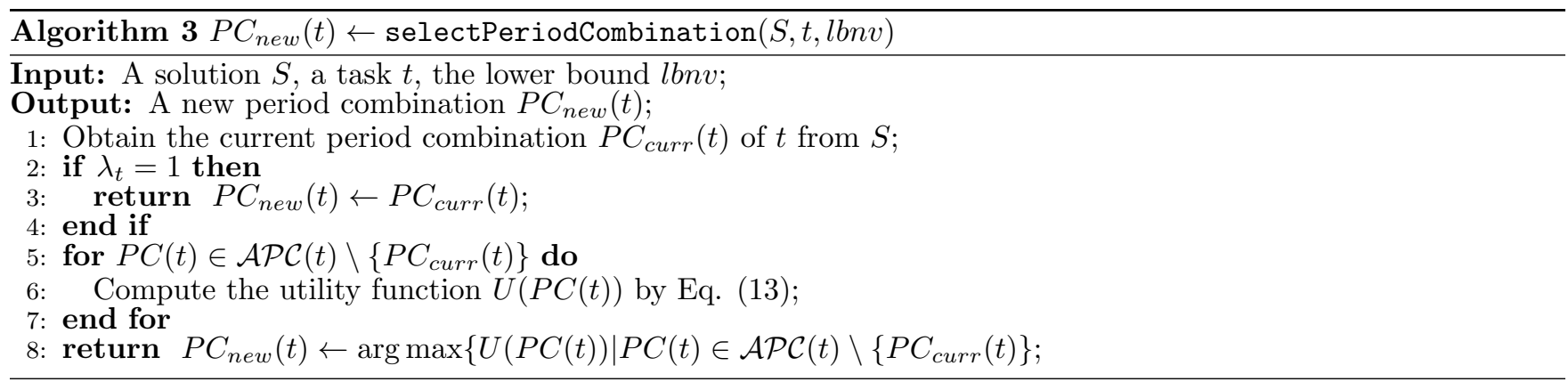

It should be noted that the capacity constraints may be violated during the route decomposition and infeasible solutions may be obtained by the RD operator. From the description of the function move(), it can be seen that the services of task $t$ are not moved in the periods of $P C_{\text {new }}(t) \cap P C_{\text {curr }}(t) \backslash\left\{p_{\text {sel }}\right\}$. In each of these periods, the accumulated demand of $t$ is updated according to $P C_{n e w}(t)$, and the load of the route containing $t$ will change. If the accumulated demand of $t$ increases, then the capacity constraints may be violated. Given the solution $S$, the total overload $\operatorname{tol}(S)$ reflects the degree of constraint violation of $S$, and is calculated as follows:

$$
\operatorname{tol}(S)=\sum_{p=1}^{n p} \sum_{k=1}^{n r_{p}} \max \left\{\operatorname{load}\left(R_{p k}\right)-Q, 0\right\}
$$

The task movement is similar to the $\lambda$-interchange operator proposed for VRP [40, which divides all the customers of a given solution into clusters and exchanges subsets between two given clusters. The size of the exchanged subsets cannot be larger than the parameter $\lambda$. The task movement proposed in this paper is similar to the $\lambda$-interchange operator, where one task is moved from one cluster to another, i.e. $\left(\lambda_{1}, \lambda_{2}\right)=(1,0)$. However, it is more complex than the $\lambda$-interchange operator, since it may move multiple services of one task to different periods.

\section{Memetic Algorithm with Route-Decomposing}

Obviously, PCARP is a kind of permutation-based optimization problems for which lots of approaches have been presented(e.g. [50], 51], 52], [53], [54]). As a relatively new population-based meta-heuristic approach, MA was firstly introduced by Moscato in 1989 [55] and effective for the permutation-based optimization problems ([56]). It was inspired by Darwinian principles of natural evolution and Dawkins' notion of memes [57], and can be regarded as a kind of hybrid genetic algorithm (GA) with local search strategies. Due to its powerful ability of searching in combinatorial solution spaces, MA has shown to be successful in many fields (e.g. [18, 19], 20], 21], 31], 34], [58, [59, 60, 61, 62]).

The proposed MARD for PCARP uses the framework of MA and focuses on three aspects. First, the RD operator is embedded between the crossover and local search operators. Second, in the initialization stage, a few high-quality initial individuals are included to help improve the convergence of population in the subsequent search process. Last but not least, during the local search, a novel operator is constructed to select the period combination of one task. In the following, we will describe the framework of MARD and each component in detail. 


\subsection{Framework of $M A R D$}

The framework of MARD for PCARP is given in Algorithm 4. Firstly, a population pop with psize nonclone

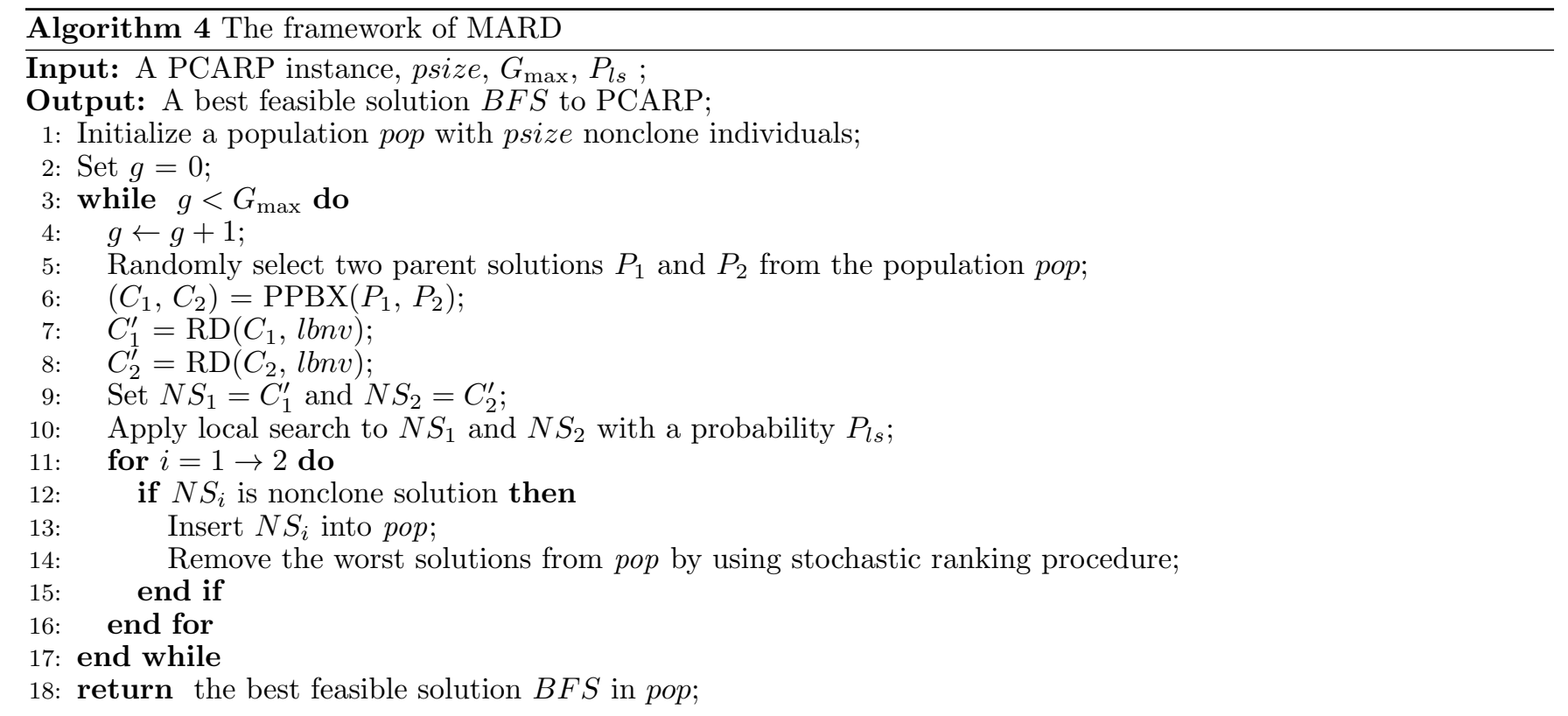

\subsection{Solution Representation and Evaluation}

Like MARM([34), we use an explicit task encoding scheme that represents a route as Eq. (1), and a solution as Eq. (2). Fig. 1 shows a simple example, in which the tasks are represented by the solid lines, and the deadheading paths are represented by the dashed lines. As mentioned before, each task is assigned two positive integer IDs, one for each direction. Then, Fig. 1 shows a PCARP solution: Period $1((0,1,2,3,0),(0,4,5,0))$ and Period $2(0,11,10,6,0)$. Given a solution $S$, the objectives $t c$ and $n v$ can be calculated according to Eq. (5) and (6) respectively, and fitness is computed by Eq. (7).

Due to the explicit task encoding scheme employed in this paper, the complexity of the fitness evaluation is $O\left(\sum_{t \in E_{R}} \eta(t)\right)=\mathrm{O}(\gamma)$, where $\gamma$ is the total number of services over the horizon.

\subsection{Population Initialization}

To ensure the diversity of pop, clones are not allowed in pop throughout the entire search process. Starting from an empty pop, the initial individuals are generated and added to pop one by one. Since the full verification 


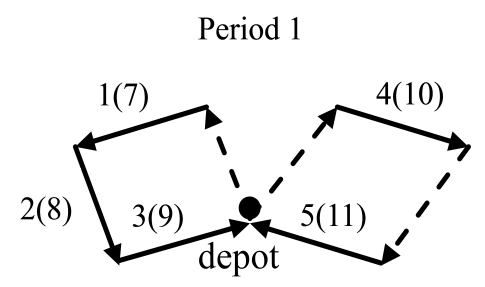

Period 2

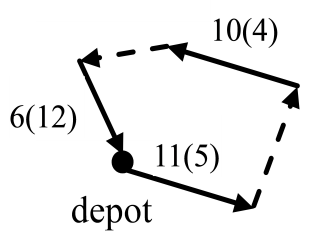

Fig.1. An simple example of the explicit task encoding scheme for PCARP solutions.

of clone individual solutions in PCARP is computationally expensive, an efficient approximate scheme is applied to two solutions $S_{1}$ and $S_{2}$, which only compares their $t c, n v$ and tol values. $S_{1}$ and $S_{2}$ are considered to be clone to each other if they have the same values in all the three properties.

It has been showed that containing a few high-quality individuals in the initial population can help improve the convergence of the population, and this initializing scheme was used by most approaches for PCARP (e.g. [31], 32, [34]). Thus, we adopt two heuristics to produce a few good solutions during initialization. To this end, the Path Scanning(PS) [6] and Best Insertion Heuristic(BIH) 30] are adopted and extended to fit the multi-period scenario. The extended heuristics are called EPS and EBIH respectively. The extension process is described as follows:

Step 1. Create $n p$ sub-lists of tasks $s l_{1}, s l_{2}, \ldots, s l_{n p}$ (one for each period), and set them to empty,

Step 2. For each task $t \in E_{R}$, select a period combination $P C(t)$ from $\mathcal{A P C}(t)$ randomly;

Step 3. For each task $t \in E_{R}$, add $t$ to corresponding period's sub-list based on the selected $P C(t)$;

Step 4. For each sub-list $s l_{p}$, apply the single-period heuristic (PS or BIH) to generate a single-period sub-solution; Step 5. Integrate all the sub-solutions obtained by Step 4 to form a complete PCARP solution.

Due to the randomness involved in PS and BIH, one can generate multiple unique solutions by the same heuristic. As a result, in the initial population, 1/6 individuals are generated by EPS, 2/3 individuals are generated by EBIH, and $1 / 6$ remaining individuals are generated randomly.

\subsection{Crossover Operator}

In this paper, a new Periodic Period-Based Crossover (PPBX) operator is developed by modifying the Periodic Linear Order Crossover (PLOX) 31. It is described in Algorithm5. In the beginning, $r$ periods are randomly chosen from the horizon. Then, $C_{1}$ inherits the routes from $P_{1}$ for the selected $r$ periods, and from $P_{2}$ for the remaining periods (lines 7 11). After that, the period combination $P C(t)$ of each task $t$ is determined on the basis of the period combination of $t$ in $P_{1}$ (lines 12 16). Briefly speaking, the new period combination includes all the periods in which $t$ is served in both $P_{1}$ and $C_{1}$, and covers the periods in which $t$ is served in both $P_{2}$ and $C_{1}$ as much as possible. Finally, for all the tasks, the services are removed from the periods that are out of the new period combination, and inserted into the missing periods in the new period combination (lines 30 38). The other offspring solution $C_{2}$ can be obtained by switching the roles of $P_{1}$ and $P_{2}$.

PPBX is similar to the Best-Cost Route Crossover (BCRC) proposed [39] in the sense that it inserts the missing tasks into the best positions. Additionally, it consists of more complex components to deal with the periodic situation, such as period selection and adjustment of period combinations of the involved tasks. 


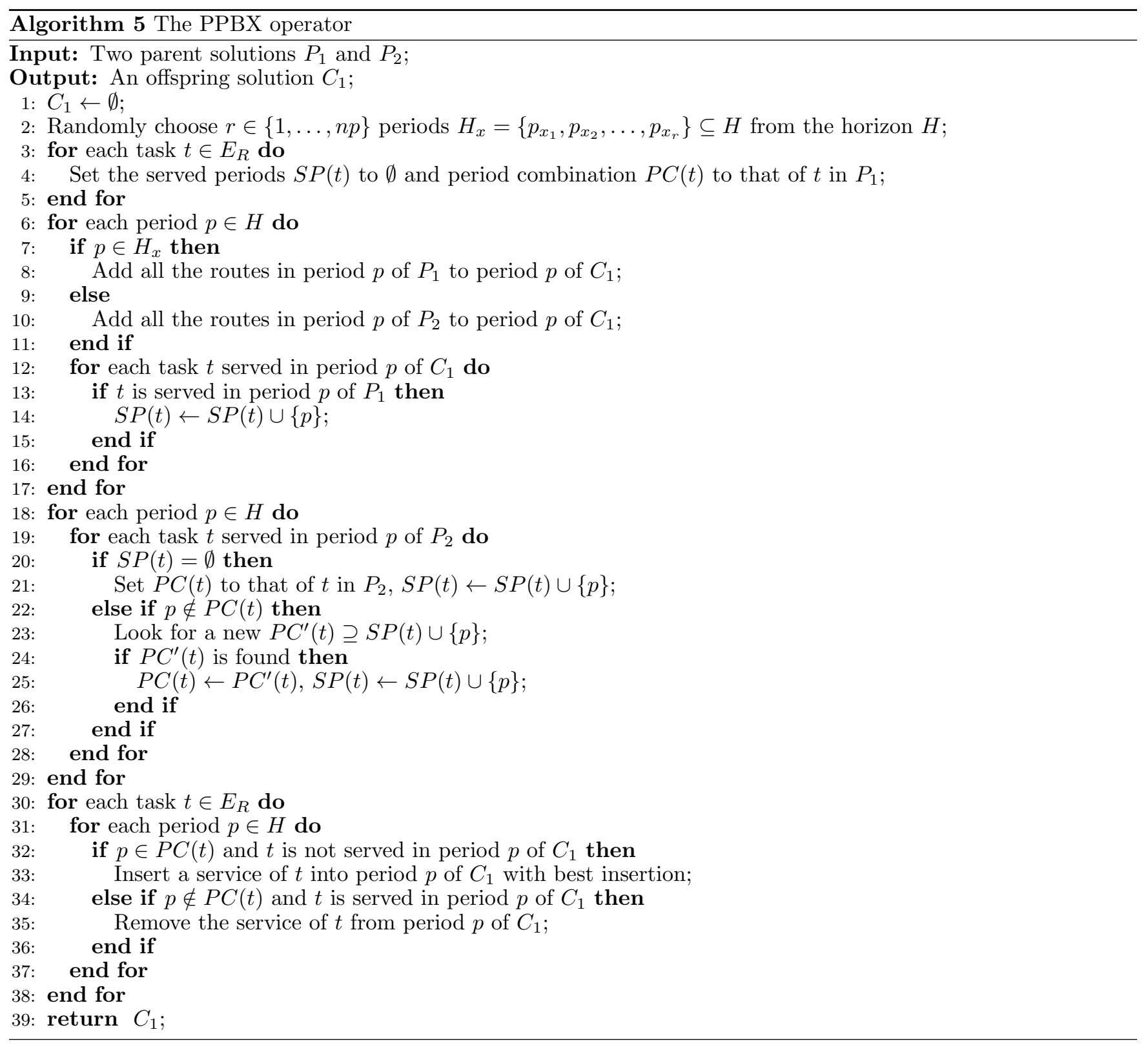

\subsection{Local Search}

The process of local search is shown in Fig. 2. It consists of two stages. The first stage is a traditional small-step local search with four traditional move operators, i.e. Single Insertion (SI), Double Insertion (DI), Swap and 2-opt, given a solution $S$ and a task $t$, each feasible period combination $P C_{\text {new }}(t) \in \mathcal{A P C}(t) \backslash\left\{P C_{\text {curr }}(t)\right\}$ is examined, and a corresponding solution $S_{\text {new }}$ is generated by removing redundant services and inserting missing services of $t$ (lines 4 10. Then, the best solution $S_{\text {best }}$ is obtained to replace the original solution $S$. In line 11 , the solutions are compared in terms of fitness value and constraint violation. That is, a solution $S_{1}$ is better than another solution $S_{2}$ if 1$) \operatorname{tol}\left(S_{1}\right)<\operatorname{tol}\left(S_{2}\right)$ or 2$) \operatorname{tol}\left(S_{1}\right)=\operatorname{tol}\left(S_{2}\right)$ and $f\left(S_{1}\right)<f\left(S_{2}\right)$.

For the RPC operator, all the possible $t$ 's are examined, and the best neighbor is selected to be the next solution. Similarly, four other solutions are produced by the SI, DI, Swap and 2-opt operators. Then, the first stage returns the best solution $S^{\prime}$ among the five solutions produced by the five operators. 

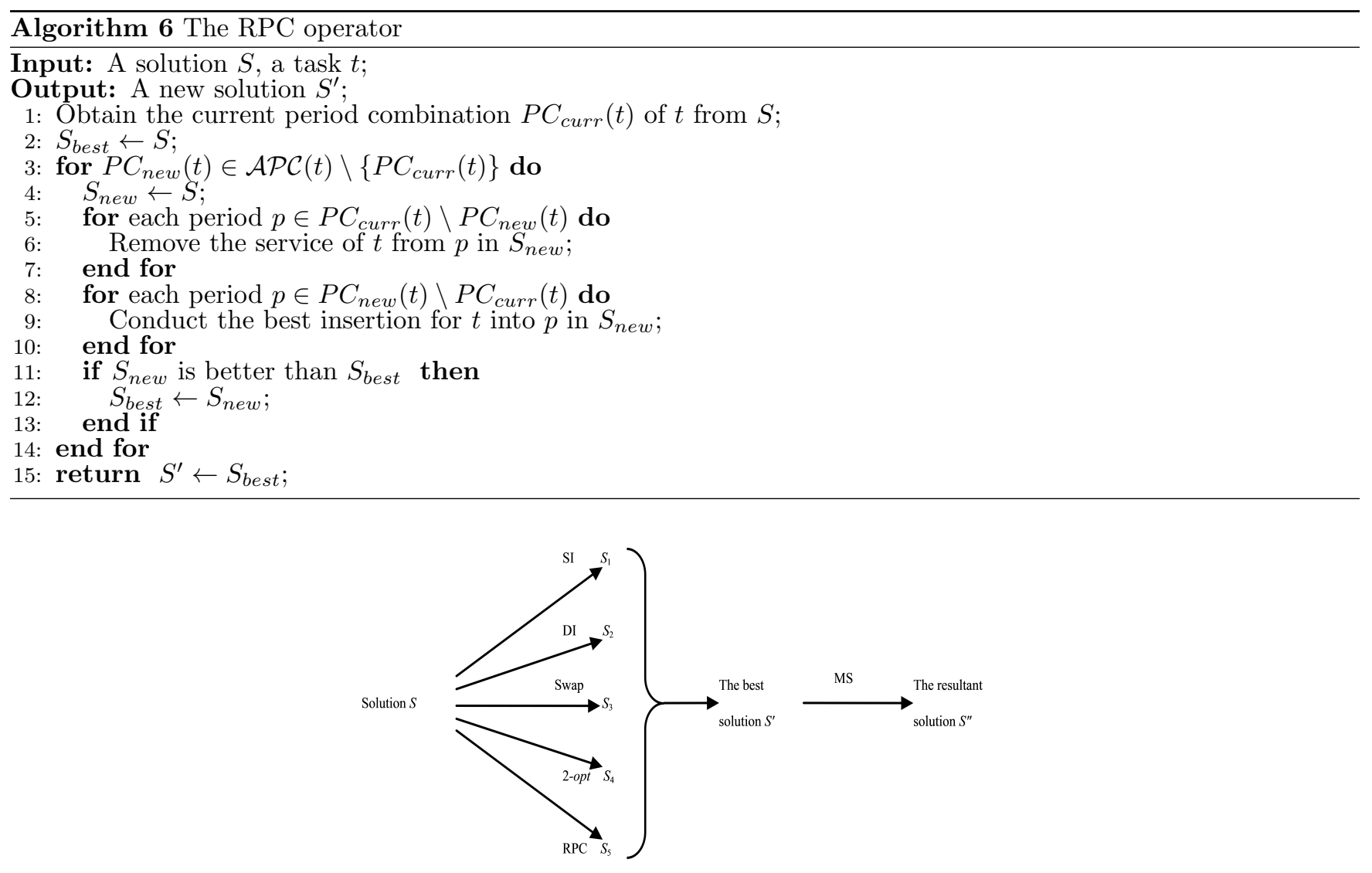

Fig.2. The local search process.

The second stage is an extended local search employing the Merge-Split (MS) operator proposed by Tang et al.

18, in an attempt to further exploit in a larger promising region. Although the MS operator has shown promise in generating high-quality solutions, it suffers from a very high computational complexity. In this paper, we improve the efficiency of the MS operator as follows: First, randomly shuffle the routes. Then, for each period, apply the MS operator to the $i$ th and $(i+1)$ th route $\left(i=1, \ldots,\left\lfloor n r_{p} / 2\right\rfloor\right)$. The resultant two routes replace the original ones if they are better. Finally, the resultant solution $S^{\prime \prime}$ is returned.

\subsection{Summary of MARD}

Finally, we summarize the characteristics of MARD by comparing with the state-of-the-art algorithms including MARM [34, LMA [31] and SS [32. The differences between MARD and other algorithms are as follows:

1) A new Route Decomposition (RD) operator is developed and embedded in MARD to improve both $n v$ and $t c$ simultaneously. The RD operator selects a route from the period with the maximum number of routes, removes it from the period, and re-inserts the tasks to other periods so that the additional cost is minimized.

2) To benefit the convergence of the search, a hybridized initialization is employed to include high-quality solutions. The PS and BIH constructive heuristics for CARP are extended to PCARP for generating the initial solutions.

3) A new crossover operator called the PPBX operator is designed to combine both the period combination and routing information of the two parents to produce the offsprings.

4) A new local search operator is designed for modifying the period combination of tasks. 


\section{Experimental Studies}

In this section, we evaluate MARD by comparing it with MARM[34, LMA [31] and SS [32] on the pgdb, pval and $p G$ test sets. In addition, the effect of the RD operator is verified by comparing MARD and its counterpart without the RD operator.

5.2. Experimental Results and Analysis

\subsubsection{Small and Medium Sized Instances}

First, we compare the algorithm on the small and median sized instances. To this end, the pgdb and pval test sets are used. The results of the average performance are summarized in Table 2 , and the detailed average results are shown in Tables 12 and 13 in the Appendix. The results of LMA, SS and MARM are obtained directly from the corresponding literatures. For LMA, three versions (DMA, PMA and IPMA) were presented in [31] and IPMA showed the best performance. Thus, in our comparison, the column LMA stands for the results of IPMA. Note that LMA and SS were run only once in the original literature. On the other hand, MARD and MARM were run for 30 times. With no guarantee on the accuracy of our reimplementation, the best we can do is to consider the results obtained from the single run of LMA and SS as their average performances. Additionally, there is no result available for LMA on the pval set from the original literature. Thus the comparison with LMA on the pval set is ignored. For each algorithm and each objective $(n v$ and $t c)$ in Table 2 , there are three columns that stand for the mean value 
over all the instances (denoted as "Mean"), the number of the best mean values among all the compared results (denoted as "NB") and the average percentage deviation from the corresponding lower bound respectively (denoted as "APD"). APD is calculated as follows:

$$
A P D=\frac{1}{N} \sum_{i=1}^{N} \frac{f_{i}-l b_{i}}{l b_{i}} \times 100
$$

where $N$ indicates the number of instances, and $f_{i}$ and $l b_{i}$ stand for the objective value and lower bound of the $i$ th instance respectively. For $n v$, the lower bound is obtained from Eq. 12. For $t c$, the lower bound is obtained from 32. All of the results can be downloaded from http://homepages.ecs.vuw.ac.nz/ yimei.

Table 2: Summary of the average results obtained by the compared algorithms on the pgdb and pval sets. For each instance, MARM and MARD were run 30 times independently. The results of LMA and SS were obtained from literature, which were obtained by running once using sophisticated parameter settings.

\begin{tabular}{|c|c|c|c|c|c|c|c|c|c|c|c|c|c|c|c|c|c|c|c|c|}
\hline \multirow[t]{3}{*}{ Test set } & \multicolumn{5}{|c|}{ LMA(IPMA) } & \multicolumn{4}{|c|}{ SS } & \multicolumn{6}{|c|}{ MARM } & \multicolumn{5}{|c|}{ MARD } \\
\hline & & $n v$ & & $t c$ & & & $n v$ & & tc & & $n v$ & & & $t c$ & & & $n v$ & & & $t c$ \\
\hline & Mean & NB APD & Mean & NB $A$ & APD & Mean & NB APD & Mean & NB APD 1 & Mean & i NB & APD & Mean & NB & $\mathrm{APD}$ & Mean & NB & APD & Mean & NB APD \\
\hline$p g d b$ & 3.13 & 8.7 & 752.22 & $\begin{array}{ll}0 & 1\end{array}$ & 12.48 & 3.35 & $14 \quad 13.84$ & 743.35 & $\begin{array}{ll}0 & 10.73\end{array}$ & 2.98 & 23 & 1.16 & 711.06 & 0 & 6.77 & 3.2 & 16 & 9.94 & 698.95 & $23 \quad 4.86$ \\
\hline pval & & & & & & 4.38 & 1025.141 & 1208.85 & $\begin{array}{ll}0 & 26.12\end{array}$ & 3.65 & 34 & 1.5 & 1129.97 & $\begin{array}{lll}7 & 0\end{array}$ & 17.72 & 3.93 & 20 & 11.79 & 1097.22 & $34 \quad 14.51$ \\
\hline
\end{tabular}

From Table 2, it can be seen that MARD performed much better than other compared algorithms overall. It achieved the best mean $n v$ values for 16/23 pgdb and 20/34 pval instances, which is much better than SS. MARM performed the best, achieving the best mean $n v$ value for all the $23 \mathrm{pgdb}$ and $34 \mathrm{pval}$ instances. However, in terms of $t c$, MARD significantly outperformed the other algorithms. It achieved the best mean $t c$ value for all the $23 p g d b$ and 34 pval instances, and its APD value is 4.86 and 14.51 for the pgdb and pval sets respectively, which is much lower than that of the other algorithms. For the $p g d b$ test set, MARD outperformed LMA (12.48) by $61.06 \%$, SS (10.73) by $54.71 \%$ and MARM (6.77) by $28.21 \%$. For the pval test set, MARD outperformed SS (26.12) by $44.45 \%$ and MARM (17.72) by $18.12 \%$.

Table 3 shows the $t$-test results between MARD and all the other algorithms on the $p g d b$ and pval datasets. For each instance, the 30 outputs of MARD and MARM are assumed to approach the normal distribution, and the single outputs of LMA and SS are considered to be the mean value. Therefore, $t$-test is considered to be applicable. In the table, W, D and L stand for Win (significantly better), Draw (no significant difference) and Lose (significantly worse), respectively. It is obvious that MARD is significantly better than both LMA and SS on most instances. Compared to both algorithms, MARD obtained the same $n v$ on most instances, and significantly better $t c$, and thus significantly better fitness value on most instances. Compared to MARM, MARD still obtained the same $n v$ and significantly better $t c$ on a fair number of instances. As a result, MARD performed significantly better than MARM on most instances (34 out of the total 57 instances) in terms of fitness. In addition, we conduct the $t$-tests with Bonferroni correction between MARD and other three compared algorithms simultaneously to see if MARD performed significantly the best, and Bonferroni correction is necessary here to do multiple comparisons. The test results on fitness are given in Table 4 in which "W" indicates the number of instances for which MARD performed significantly better than all the other algorithms. "D" stands for the number of instances for which MARD performed either significantly the same as at least one algorithm, and significantly better than the remaining algorithms. "L" is the number of instances for which MARD performed significantly worse than at least one of the compared algorithms. From the table, it can be seen that MARD achieved significantly better fitness values than all the other compared algorithms on more than half (14 out of $23 \mathrm{pgdb}$ and 19 out of $34 \mathrm{pval}$ ) instances. 
Table 3: The results of the $t$-tests with signficance probability of $95 \%$ between MARD and the other compares algorithms on the pgdb and pval instances.

\begin{tabular}{|c|c|c|c|c|c|c|c|c|c|c|c|c|c|c|c|c|c|c|c|c|c|c|c|c|c|c|c|}
\hline \multirow{3}{*}{ Test set } & \multicolumn{9}{|c|}{ MARD and LMA } & \multicolumn{9}{|c|}{ MARD and SS } & \multicolumn{9}{|c|}{ MARD and MARM } \\
\hline & \multicolumn{3}{|c|}{$n v$} & \multicolumn{3}{|c|}{$t c$} & \multicolumn{3}{|c|}{ fitness } & \multicolumn{3}{|c|}{$n v$} & \multicolumn{3}{|c|}{$t c$} & \multicolumn{3}{|c|}{ fitness } & \multicolumn{3}{|c|}{$n v$} & \multicolumn{3}{|c|}{$t c$} & \multicolumn{3}{|c|}{ fitness } \\
\hline & $\mathrm{W}$ & $\mathrm{D}$ & $\mathrm{L}$ & $\mathrm{W}$ & $\mathrm{D}$ & $\mathrm{L}$ & $\mathrm{W}$ & $\mathrm{D}$ & $\mathrm{L}$ & $\mathrm{W}$ & $\mathrm{D}$ & $\mathrm{L}$ & $\mathrm{W}$ & $\mathrm{D}$ & $\mathrm{L}$ & $\mathrm{W}$ & $\mathrm{D}$ & $\mathrm{L}$ & $\mathrm{W}$ & $\mathrm{D}$ & $\mathrm{L}$ & $\mathrm{W}$ & $\mathrm{D}$ & $\mathrm{L}$ & $\mathrm{W}$ & $\mathrm{D}$ & $\mathrm{L}$ \\
\hline$p g d b$ & 1 & 18 & 4 & 23 & 0 & 0 & 18 & 1 & 4 & 6 & 17 & 0 & 23 & 0 & 0 & 23 & 0 & 0 & 0 & 15 & 8 & 23 & 0 & 0 & 14 & 1 & 8 \\
\hline pval & & & & & & & & & & 18 & 16 & 0 & 34 & 0 & 0 & 31 & 3 & 0 & 0 & 20 & 14 & 33 & 1 & 0 & 20 & 0 & 14 \\
\hline Overall & 1 & 18 & 4 & 23 & 0 & 0 & 18 & 1 & 4 & 24 & 33 & 0 & 57 & 0 & 0 & 54 & 3 & 0 & 0 & 35 & 22 & 56 & 1 & 0 & 34 & 1 & 22 \\
\hline
\end{tabular}

Table 4: The results of the $t$-tests with Bonferroni correction (significance probability of $95 \%$ ) between MARD and the other compared algorithms on the pgdb and pval instances.

\begin{tabular}{ccccccccc}
\hline \multirow{2}{*}{ Test set } & \multicolumn{3}{c}{ fitness } & & \multicolumn{3}{c}{ fitness } \\
\cline { 2 - 4 } \cline { 7 - 9 } & W & D & L & & & W & D & L \\
\hline$p g d b$ & 14 & 2 & 7 & pval & 19 & 1 & 14 \\
\hline
\end{tabular}

Table 5 shows the summary of the best results obtained by the compared algorithms on the pgdb and pval test sets, and Tables 14 and 15 show the detailed results. Note that the results of LMA and SS were obtained by a single run with a sophisticated setting, thus we still keep them in the tables for reference.

Table 5: Overall comparison of the best results obtained by the compared algorithms on the test sets pgdb and pval. MARM and MARD were run for 30 times independently, and LMA and SS were run once using sophisticated parameter settings.

\begin{tabular}{|c|c|c|c|c|c|c|c|c|c|c|c|c|c|c|c|c|c|c|c|c|c|c|c|c|}
\hline \multirow[t]{3}{*}{ Test set } & \multicolumn{6}{|c|}{ LMA(IPMA) } & \multicolumn{6}{|c|}{ SS } & \multicolumn{6}{|c|}{ MARM } & \multicolumn{6}{|c|}{ MARD } \\
\hline & \multicolumn{3}{|c|}{$n v$} & \multicolumn{3}{|c|}{$t c$} & \multicolumn{3}{|c|}{$n v$} & \multicolumn{3}{|c|}{$t c$} & \multicolumn{3}{|c|}{$n v$} & \multicolumn{3}{|c|}{$t c$} & \multicolumn{3}{|c|}{$n v$} & \multicolumn{3}{|c|}{$t c$} \\
\hline & Mean & NB & $A P D$ & Mean & $\mathrm{NB}$ & $\mathrm{APD}$ & Mean & $\mathrm{NB}$ & $\mathrm{APD}$ & Mean & NB & $\mathrm{APD}$ & Mean & $\mathrm{NB}$ & $\mathrm{APD}$ & Mean & $\mathrm{NB}$ & $\mathrm{APD}$ & Mean & $\mathrm{NB}$ & $\mathrm{APD}$ & Mean & NB & $\mathrm{APD}$ \\
\hline$p g d b$ & 3.13 & 19 & 8.7 & 752.22 & 0 & 12.48 & 3.35 & 14 & 13.84 & 743.35 & 0 & 10.73 & 2.96 & 23 & 0 & 696.91 & 2 & 4.75 & 3.13 & 19 & 8.7 & 688.13 & 23 & 3.43 \\
\hline pval & & & & & & & 4.38 & 10 & 25.14 & 1208.85 & 0 & 26.12 & 3.62 & 34 & 0 & 1106.5 & 3 & 15.14 & 3.74 & 30 & 5.88 & 1083.18 & 34 & 12.8 \\
\hline
\end{tabular}

From Table 5, it is clear that MARD performed competitively in terms of both $n v$ and $t c$ in the best case. It achieved the best $n v$ value on $19 \mathrm{pgdb}$ and $30 \mathrm{pval}$ instances, which is slightly worse than MARM, the same as LMA and much better than SS. In terms of $t c$, MARD achieved the best results on all the 57 instances, and further updated the best-known results for $19 \mathrm{pgdb}$ and 31 pval instances (see Tables 14 and 15). On the other hand, the APD values of $n v$ obtained by MARD on test sets $p g d b$ and pval are 8.7 and 5.88, they are still comparable with those achieved by the other algorithms like the compared algorithms' average performance. However, in terms of $t c$, the APD value of the best $t c$ obtained by MARD on test set $p g d b$ is 3.43, which is better than that of LMA (12.48) by $73.24 \%$, SS (10.73) by $68.03 \%$ and MARM (4.75) by $17.03 \%$. For the pval test set, the APD value of the best $t c$ obtained by MARD is 12.8, which is better than that of SS (26.12) by $51.00 \%$ and MARM (15.14) by $15.46 \%$. Overall, in the best case, MARD outperformed all the other algorithms and the best-known results.

Table 6 shows the average running time (in seconds) of the compared algorithms. Note that the running time depends on various factors such as CPU frequency, RAM, programming language, compiler, etc. It is hardly possible to make a fair comparison between the running times on different platforms. However, we can still get a rough idea by normalizing the running times with respect to the CPU frequency. MARD was run on Intel(R) Core(TM) i3-3240 M 3.40 GHz. MARM was run on Intel(R) Xeon(R) E5335 2.00 GHz. LMA and SS were run on Pentium 4 at $1.40 \mathrm{GHz}$ and $2.40 \mathrm{GHz}$, respectively. Therefore, the running times of MARM, LMA and SS are multiplied by 
2/3.4, 1.4/3.4 and 2.4/3.4, respectively. The table shows that MARD is slower than MARM and SS, while faster than LMA. The high complexity of MARD is mainly due to the RD operator, which enumerates all the possible positions when inserting each task service. In addition, during the local search MARD employs more operators than the other algorithms (e.g. MS and RPC). How to improve the efficiency of MARD by identifying and removing possible redundant computations will be our future direction. Some studies in memetic computing take advantage of the domain knowledge (represented as the so-called memes) learned from solving related problems in the past and/or online learning. For instance, Feng et al. [41] proposed to use the promising solution structure learned from solving CVRP to initialise better solutions for CARP, and vice versa. Chen et al. 64 proposed to use memes storing different local search operators, and developed an algorithm to learn when to use which local search operator during the search process. To improve efficiency, we can employ similar ideas to learn the domain knowledge to focus on the promising regions in the search space.

Table 6: Average running time (in seconds) of the compared algorithms, normalized by CPU frequency. The running times of LMA, SS and MARM are multiplied by $2 / 3.4,1.4 / 3.4$ and $2.4 / 3.4$, respectively.

\begin{tabular}{ccccc}
\hline Test set & LMA(IPMA) & SS & MARM & MARD \\
\hline pgdb & 192.2 & 78.8 & 7.2 & 82 \\
pval & & 312.6 & 30.9 & 358.6 \\
\hline
\end{tabular}

Since the RD operator is the main contribution of this work, it is important to verify its efficacy in MARD. To this end, we remove the RD operator (lines 7 and 8 in Algorithm 4 from MARD, and compare this variant, called $\mathrm{MA}^{*}$, against MARD on the $p g d b$ and pval instances over 30 independent runs. The summarized average results are given in Table 7. From the table, it can be seen that MARD achieved much smaller $n v$ value than MA*, while the $t c$ value is nearly the same. This is consistent with our expectation, which suggests that the RD operator can reduce $n v$ while not much deteriorating $t c$.

Table 7: Summary of the average results obtained by 30 independent runs of MARD and $\mathrm{MA}^{*}$ on the test sets $p g d b$ and pval.

\begin{tabular}{cccccc}
\hline \multirow{2}{*}{ Test set } & \multicolumn{2}{c}{$\mathrm{MA}^{*}$ (without RD) } & & \multicolumn{2}{c}{ MARD } \\
\cline { 2 - 3 } \cline { 5 - 6 } \cline { 5 - 6 } & $n v$ & $t c$ & & $n v$ & $t c$ \\
\hline pgdb & $3.35 \pm 0.11$ & $699.79 \pm 5.82$ & & $3.20 \pm 0.10$ & $698.95 \pm 5.23$ \\
pval & $4.42 \pm 0.20$ & $1092.92 \pm 10.02$ & & $3.93 \pm 0.12$ & $1097.22 \pm 11.42$ \\
Overall & $3.89 \pm 0.16$ & $896.36 \pm 7.92$ & & $3.56 \pm 0.11$ & $898.09 \pm 8.33$ \\
\hline
\end{tabular}

More specifically, Table 8 gives the numbers of Win, Draw and Lose instances of MARD against MA* when conducting the $t$-test under the confidence probability of $95 \%$. It can be seen that MARD improved the solutions on the test sets greatly in contrast with $\mathrm{MA}^{*}$ in both objectives. In term of $n v$, MARD is significantly better on 10 $p g d b$ and 24 pval instances, while never performed significantly worse. In term of $t c$, MARD and MA* performed statistically the same on $17 \mathrm{pgdb}$ and 23 pval instances. Note that MARD was significantly outperformed by MA* on 10 pval instances. However, MARD achieved significantly better $n v$ values on most of these instances. As a result, MARD still showed significantly better performance than MA* in terms of fitness.

\subsubsection{Large-Scale Instances}

In addition to the small and medium sized instances, we also compared MARD with MARM, LMA and SS on the large $p G$ instances to evaluate its scalability. The results were obtained directly from 34. Note that LMA 
Table 8: The statistical test results obtained by 30 independent runs of MARD and $\mathrm{MA}^{*}$ on the test sets $p g d b$ and pval.

\begin{tabular}{|c|c|c|c|c|c|c|c|c|c|}
\hline \multirow[t]{2}{*}{ Test Set } & \multicolumn{3}{|c|}{$n v$} & \multicolumn{3}{|c|}{$t c$} & \multicolumn{3}{|c|}{ fitness } \\
\hline & $\mathrm{W}$ & D & L & $\mathrm{W}$ & $\mathrm{D}$ & $\mathrm{L}$ & $\mathrm{W}$ & $\mathrm{D}$ & L \\
\hline$p g d b$ & 10 & 13 & 0 & 4 & 17 & 2 & 11 & 12 & 0 \\
\hline pval & 24 & 10 & 0 & 1 & 23 & 10 & 24 & 9 & 1 \\
\hline Overall & 34 & 23 & 0 & 5 & 40 & 12 & 35 & 21 & 1 \\
\hline
\end{tabular}

and SS were not tested on the $p G$ set in their original works [31, 32, but reimplemented in [34. Thanks to the clear description of the algorithms in the original literatures, we believe that the reimplemented version will have statistically comparable behaviour with the original ones. The best and average results (with the $t$-test under confidence probability of 95\%) of the compared algorithms are presented in Tables 9 and 10 . The summarized statistical test results are shown in Table 11. Being a new PCARP test set, the lower bounds of $t c$ are unavailable for the $p G$ test set.

Table 9: Best results of the composed algorithms on $p G$. The results in bold face are best and the new best results achieved by our MARD are showed with "*”.

\begin{tabular}{|c|c|c|c|c|c|c|c|c|c|c|c|c|}
\hline \multirow[t]{2}{*}{ Name } & \multirow[t]{2}{*}{$|V|$} & \multirow[t]{2}{*}{$|E|$} & \multirow[t]{2}{*}{$\gamma$} & \multirow[t]{2}{*}{ LBV } & \multicolumn{2}{|c|}{ LMA } & \multicolumn{2}{|r|}{ SS } & \multicolumn{2}{|c|}{ MARM } & \multicolumn{2}{|c|}{ MARD } \\
\hline & & & & & $n v$ & $t c$ & $n v$ & $t c$ & $n v$ & $t c$ & $n v$ & $t c$ \\
\hline G1-A & 255 & 347 & 1062 & 10 & 11 & 3623482 & 12 & 3513307 & 10 & 3678504 & 10 & $3106420 *$ \\
\hline G1-B & 255 & 347 & 1062 & 12 & 14 & 3874996 & 15 & 3808003 & 13 & 3947359 & 13 & $3437158^{*}$ \\
\hline G1-C & 255 & 347 & 1062 & 11 & 13 & 3690919 & 14 & 3710681 & 11 & 3799614 & 12 & $3260348^{*}$ \\
\hline G2-A & 255 & 375 & 1138 & 11 & 12 & 3820365 & 14 & 3814253 & 11 & 3923292 & 11 & $3383565 *$ \\
\hline G2-B & 255 & 375 & 1138 & 13 & 15 & 4118258 & 16 & 4108672 & 14 & 4235707 & 14 & $3656182^{*}$ \\
\hline G2-C & 255 & 375 & 1138 & 11 & 14 & 4019023 & 15 & 3949113 & 13 & 4103535 & $12^{*}$ & $3501008^{*}$ \\
\hline Mean & & & & 11.3 & 13.2 & 3857840.5 & 14.3 & 3817338.2 & 12.0 & 3948001.8 & 12.0 & 3390780.2 \\
\hline
\end{tabular}

From Table 9, MARD is superior to LMA and SS on all the $6 p G$ instances and obtained the same number of best $n v$ as MARM. In particular, MARD achieved solutions with significantly smaller tc than that of all other composed algorithms on all the 6 instances, Although on instance G1-C, $n v$ obtained by MARD is worse than that of MARM, fitness achieved by MARD is better than that of MARM ( $\alpha=100000$ in the experiment). Thus, MARD achieved the best solutions on all $p G$ instances.

In terms of average performance, Tables 10 and 11 show that MARD showed significantly better performance than both LMA and SS on all the $6 p G$ instances in terms of $n v, t c$ and fitness. When comparing to MARM, MARD performed significantly better on 3 instances in terms of both $n v$ and $t c$. On the remaining 3 instances, MARD achieved the same $n v$ and significantly better $t c$, and thus significantly better fitness. In addition, we conducted the $t$-tests with Bonferroni correction between MARD and other three compared algorithms, and the results show that MARD obtained significant better fitness than all the other algorithms on all the $p G$ instances.

In summary, MARD showed significant advantage over all the compared state-of-the-art algorithms on the largescale $p G$ instances. That is, MARD has a good scalability in terms of solution quality, i.e. its advantage becomes more obvious as the problem size increases. This may be because when the problem becomes larger and more complicated, the solution repairing (reinsertion of missing tasks) needs to be more sophisticated to improve the convergence of the population. In this sense, the RD operator has a strong advantage over the previous operators. 
Table 10: Average results obtained by 30 independent runs of the composed algorithms on $p G$. The results with "†" obtained by one of the compared algorithm are significantly better than that of all the others (with confidence probability of $95 \%$ )

\begin{tabular}{|c|c|c|c|c|c|c|c|c|}
\hline \multirow[t]{2}{*}{ Name } & \multicolumn{2}{|r|}{ LMA } & \multicolumn{2}{|r|}{ SS } & \multicolumn{2}{|r|}{ MARM } & \multicolumn{2}{|r|}{ MARD } \\
\hline & $n v$ & $t c$ & $n v$ & $t c$ & $n v$ & $t c$ & $n v$ & $t c$ \\
\hline G1-A & $12.1 \pm 0.6$ & $3846199.2 \pm 204563.0$ & $13.1 \pm 0.4$ & $4191076.8 \pm 589288.5$ & $11.0 \pm 0.4$ & $3758286.1 \pm 48305.9$ & $10.9 \pm 0.4$ & $3157654.0 \pm 37669.3^{\dagger}$ \\
\hline G1-B & $15.1 \pm 0.8$ & $4170835.4 \pm 223624.0$ & $16.3 \pm 0.6$ & $4319454.0 \pm 457226.7$ & $14.0 \pm 0.7$ & $4093805.0 \pm 67843.8$ & $13.6 \pm 0.6$ & $3488414.7 \pm 34891.7^{\dagger}$ \\
\hline G1-C & $13.6 \pm 0.6$ & $4034616.1 \pm 185334.1$ & $14.8 \pm 0.4$ & $4230602.5 \pm 593157.0$ & $12.3 \pm 0.7$ & $3935364.7 \pm 66648.1$ & $12.4 \pm 0.5$ & $3315679.9 \pm 36691.5^{\dagger}$ \\
\hline G2-A & $13.5 \pm 0.8$ & $4189723.8 \pm 233001.0$ & $14.6 \pm 0.5$ & $4306027.1 \pm 504355.2$ & $12.3 \pm 0.5$ & $4051017.5 \pm 56278.0$ & $12.0 \pm 0.4^{\dagger}$ & $3414931.2 \pm 29000.3^{\dagger}$ \\
\hline G2-B & $16.3 \pm 0.8$ & $4515713.9 \pm 273187.1$ & $17.6 \pm 0.6$ & $4584540.6 \pm 511354.6$ & $15.0 \pm 0.8$ & $4411075.3 \pm 81881.3$ & $14.5 \pm 0.5^{\dagger}$ & $3720668.8 \pm 40338.2^{\dagger}$ \\
\hline $\mathrm{G} 2-\mathrm{C}$ & $14.6 \pm 0.6$ & $4362438.9 \pm 299696.1$ & $15.8 \pm 0.5$ & $4493359.9 \pm 586055.1$ & $13.4 \pm 0.5$ & $4210976.6 \pm 55288.2$ & $13.2 \pm 0.5$ & $3531334.8 \pm 34262.3^{\dagger}$ \\
\hline Mean & 14.2 & 4186587.9 & 15.4 & 4354176.8 & 13 & 4076754.2 & 12.8 & 3438113.9 \\
\hline
\end{tabular}

Table 11: The statistical test results obtained by 30 independent runs of MARD and other compared algorithms on the $p G$ set.

\begin{tabular}{|c|c|c|c|c|c|c|c|c|c|}
\hline \multirow[t]{2}{*}{ Item } & \multicolumn{3}{|c|}{ MARD and LMA } & \multicolumn{3}{|c|}{ MARD and SS } & \multicolumn{3}{|c|}{ MARD and MARM } \\
\hline & $\mathrm{W}$ & $\mathrm{D}$ & $\mathrm{L}$ & $\mathrm{W}$ & $\mathrm{D}$ & $\mathrm{L}$ & $\mathrm{W}$ & $\mathrm{D}$ & $\mathrm{L}$ \\
\hline$n v$ & 6 & 0 & 0 & 6 & 0 & 0 & 3 & 3 & 0 \\
\hline$t c$ & 6 & 0 & 0 & 6 & 0 & 0 & 6 & 0 & 0 \\
\hline fitness & 6 & 0 & 0 & 6 & 0 & 0 & 6 & 0 & 0 \\
\hline
\end{tabular}

\section{Conclusions and Future Work}

In this paper, we investigate an extended version of CARP, i.e., PCARP, which is considered over the horizon with multiply periods. PCARP is closer to practical applications (e.g. waste collection) than CARP. In addition,

\section{Acknowledgements}

This work was partially supported by the Natural Science Key Research Project for Higher Education Institutions of Anhui Province (No. KJ2016A438, No. KJ2014A140, No. KJ2013A177) and the Anhui Provincial Natural Science Foundation (No. 1408085MF131). Yi Mei is supported by the Marsden Funds of New Zealand (VUW1209), and the University Research Funds of Victoria University of Wellington (No. 203936/3337). 


\section{References}

[1] B. Golden, R. Wong, Capacitated arc routing problems, Networks 11 (3) (1981) 305-315.

[2] M. Yaghini, M. Momeni, M. Sarmadi, A simplex-based simulated annealing algorithm for node-arc capacitated multicommodity network design, Appl. Soft Comput. 12 (2012) 2997-3003.

[3] J. Kratica, M. Milanović, Z. Stanimirović, D. Tošić, An evolutionary-based approach for solving a capacitated hub location problem, Appl. Soft Comput. 11 (2011) 1858-1866.

[4] R. Hirabayashi, Y. Saruwatari, Tour construction algorithm for the capacitated arc routing-problems, AsiaPacific J. Oper. Res. 9 (2) (1992) 155-175.

[5] P. Lacomme, C. Prins, W. Ramdane-Chérif, Competitive memetic algorithms for arc routing problems, Annals of Operations Research 131 (1-4) (2004) 159-185.

[6] B. Golden, J. DeArmon, E. Baker, Computational experiments with algorithms for a class of routing problems, Comput. Oper. Res. 10 (1) (1983) 47-59.

[7] G. Ulusoy, The fleet size and mix problem for capacitated arc routing, Eur. J. Oper. Res. 22 (3) (1985) 329-337.

[8] W. Pearn, Approximate solutions for the capacitated arc routing problem, Comput. Oper. Res. 16 (6) (1989) 589-600.

[9] W. Pearn, Augment-insert algorithms for the capacitated arc routing problem, Comput. Oper. Res. 18 (2) (1991) 189-198.

[10] A. Hertz, G. Laporte, M. Mittaz, A tabu search heuristic for the capacitated arc routing problem, Oper. Res. 48 (1) (2000) 129-135.

[11] J. Brandão, R. Eglese, A deterministic tabu search algorithm for the capacitated arc routing problem, Comput. Oper. Res. 35 (4) (2008) 1112-1126.

[12] Y. Mei, K. Tang, X. Yao, A global repair operator for capacitated arc routing problem, IEEE Trans. Syst., Man, Cybern. B, Cybern. 39 (3) (2009) 723-734.

[13] A. Hertz, M. Mittaz, A variable neighborhood descent algorithm for the undirected capacitated arc routing problem, Transp. Sci. 35 (4) (2001) 425-434.

[14] M. Polacek, K. Doerner, R. Hartl, et al., A variable neighborhood search for the capacitated arc routing problem with intermediate facilities, J. Heuristics 14 (5) (2008) 405-423.

[15] P. Beullens, L. Muyldermans, D. Cattrysse, et al., A guided local search heuristic for the capacitated arc routing problem, Eur. J. Oper. Res. 147 (3) (2003) 629-643.

[16] P. Repoussis, C. Tarantilis, G. Ioannou, Arc-guided evolutionary algorithm for the vehicle routing problem with time windows, IEEE Trans. Evol. Comput. 13 (3) (2009) 624-647.

[17] Y. Mei, K. Tang, X. Yao, Improved memetic algorithm for capacitated arc routing problem, in: Proceedings of the 2009 IEEE Congress on Evolutionary Computation (CEC2009), IEEE Press, 18-21 May, 2009, pp. 16991706. 
[18] K. Tang, Y. Mei, X. Yao, Memetic algorithm with extended neighborhood search for capacitated arc routing problems, IEEE Trans. Evol. Comput. 13 (5) (2009) 1151-1166.

[19] Y. Mei, K. Tang, X. Yao, Decomposition-based memetic algorithm for multiobjective capacitated arc routing problem, IEEE Trans. Evol. Comput. 15 (2) (2011) 151-165.

[20] R. Shang, J. Wang, L. Jiao, et al., An improved decomposition-based memetic algorithm for multi-objective capacitated arc routing problem, Appl. Soft Comput. 19 (2014) 343-361.

[21] Z. Wang, H. Jin, M. Tian, Rank-based memetic algorithm for capacitated arc routing problems, Appl. Soft Comput. 37 (2015) 572-584.

[22] P. Lacomme, C. Prins, M. Sevaux, A genetic algorithm for a bi-objective capacitated arc routing problem, Comput. Oper. Res. 33 (12) (2006) 3473-3493.

[23] Y. Mei, X. Li, X. Yao, Cooperative coevolution with route distance grouping for large-scale capacitated arc routing problems, IEEE Trans. Evol. Comput. 18 (3) (2014) 435-449.

[24] R. Eglese, Routing winter gritting vehicles, Discrete Appl. Math. 48 (3) (1994) 231-244.

[25] K. Doerner, R. Hartl, V. Maniezzo, et al., Applying ant colony optimization to the capacitated arc routing problem, in: Proc. Ant Colony Optimization Swarm Intell., Lecture Notes in Computer Science 3172, Springer, 2004, pp. 420-421.

[26] A. Corberán, C. Prins, Recent results on arc routing problems: An annotated bibliography, Networks 56 (1) (2010) 50-69.

[27] P. Lacomme, C. Prins, W. Ramdane-Chérif, Evolutionary algorithms for multiperiod arc routing problems, in: Proc. 9th Int. Conf. Inf. Process. Manage. Uncertainty Knowl.-Based Syst., 2002, pp. 845-852.

[28] F. Chu, N. Labadi, C. Prins, Lower bounds for the periodic capacitated arc routing problem, in: Oral Communication, $2^{\text {nd }}$ International Workshop on Freight Transportation and Logistics (Odysseus 2003), Palermo, Italy, 2003.

[29] F. Chu, N. Labadi, C. Prins, The periodic capacitated arc routing problem: Linear programming model, metaheuristic and lower bounds, J. Syst. Sci. Syst. Eng. 13 (4) (2004) 423-435.

[30] F. Chu, N. Labadi, C. Prins, Heuristics for the periodic capacitated arc routing problem, J. Intell. Manuf. 16 (2) (2005) 243-251.

[31] P. Lacomme, C. Prins, W. Ramdane-Chérif, Evolutionary algorithms for periodic arc routing problems, Eur. J. Oper. Res. 165 (2) (2005) 535-553.

[32] F. Chu, N. Labadi, C. Prins, A scatter search for the periodic capacitated arc routing problem, Eur. J. Oper. Res. 169 (2) (2006) 586-605.

[33] A. Kansou, A. Yassine, Ant colony system for the periodic capacitated arc routing problem, in: Proc. 2009 International Network Optimization Conference, 2009, pp. 1-7.

[34] Y. Mei, K. Tang, X. Yao, A memetic algorithm for periodic capacitated arc routing problem, IEEE Trans. Syst. Man Cybern. B 41 (6) (2011) 1654-1667. 
[35] Á. Corberán, G. Laporte, Arc routing: problems, methods, and applications, Vol. 20, SIAM - Society For Industrial And Applied Mathematics, 2015.

[36] I. Monroy, C. Amaya, A. Langevin, The periodic capacitated arc routing problem with irregular services, Discrete Appl. Math. 161 (4) (2013) 691-701.

[37] S. Huang, T. Lin, Using ant colony optimization to solve periodic arc routing problem with refill points, Journal of Industrial and Production Engineering 31 (7) (2014) 441-451.

[38] J.-P. Riquelme-Rodríguez, M. Gamache, A. Langevin, Periodic capacitated arc-routing problem with inventory constraints, J. Oper. Res. Soc. 65 (12) (2014) 1840-1852.

[39] F. Hanshar, B. Ombuki-Berman, Dynamic vehicle routing using genetic algorithms, Appl. Intell. 27 (1) (2007) 89-99.

[40] I. Osman, N. Christofides, Capacitated clustering problems by hybrid simulated annealing and tabu search, Int. T. Oper. Res. 1 (3) (1994) 317-336.

[41] L. Feng, Y. Ong, M. Lim, et al., Memetic search with inter-domain learning: A realization between cvrp and carp, IEEE Trans. Evol. Comput. 19 (5) (2014) 1-15.

[42] J. Luo, X. Li, M. Chen, et al., A novel hybrid shuffled frog leaping algorithm for vehicle routing problem with time windows, Inf. Sci. 316 (2015) 266-292.

[43] L. Tan, F. Lin, H. Wang, Adaptive comprehensive learning bacterial foraging optimization and its application on vehicle routing problem with time windows, Neurocomputing 151 (2015) 1208-1215.

[44] M. Gaudioso, G. Paletta, A heuristic for the periodic vehicle routing problem, Transp. Sci. 26 (2) (1992) 86-92.

[45] E. Angelelli, G. Speranza, The periodic vehicle routing problem with intermediate facilities, Eur. J. Oper. Res. 137 (2) (2002) 233-247.

[46] P. Francis, K. Smilowitz, Modeling techniques for periodic vehicle routing problems, Transp. Res. Part B: Methodol. 40 (10) (2006) 872-884.

[47] F. Alonso, M. Alvarez, J. Beasley, A tabu search algorithm for the periodic vehicle routing problem with multiple vehicle trips and accessibility restrictions, J. Oper. Res. Soc. 59 (7) (2008) 963-976.

[48] H. Longo, M. de Aragão, E. Uchoa, Solving capacitated arc routing problems using a transformation to the CVRP, Comput. Oper. Res. 33 (6) (2006) 1823-1837.

[49] E. Dijkstra, A note on two problems in connection with graphs, Numer. Math., Vol. 1, 1959, pp. 269-271.

[50] Z. Xiao, Z. Ming, A method of workflow scheduling based on colored petri nets, Data Knowl. Eng. 70 (2) (2011) 230-247.

[51] Z. Chen, M. Qiu, Z. Ming, et al., Clustering scheduling for hardware tasks in reconfigurable computing systems, J. Syst. Architect. 59 (10) (2013) 1424-1432.

[52] J. Li, M. Qiu, J. Niu, et al., Thermal-aware task scheduling in 3d chip multiprocessor with real-time constrained workloads, ACM Trans. Embedded Comput.Syst. 12 (2) (2013) 24:1-24:22. 
[53] H. Luo, J. Fang, G. Huang, Real-time scheduling for hybrid flowshop in ubiquitous manufacturing environment, Computers \& Industrial Engineering 84 (2015) 12-23.

[55] P. Moscato, On evolution, search, optimization, genetic algorithms and martial arts: Towards memetic algorithms, CalTech, Pasadena, CA, CalTech Concurrent Computation Program Rep. 826, 1989.

[56] Z. Zhu, J. Xiao, J.-Q. Li, et al., Global path planning of wheeled robots using multi-objective memetic algorithms, Integr. Comput. Aided Eng. 22 (4) (2015) 387-404.

[57] R. Dawkins, The Selfish Gene, Oxford, U.K.: Oxford Univ., 1989.

[58] Y. Ong, Artificial intelligence technologies in complex engineering design, Ph.D. dissertation, Sch. Eng. Sci., Univ. Southampton, Southampton, U.K., 2002.

[59] J. Smith, Co-evolving memetic algorithms: A learning approach to robust scalable optimization, in: IEEE

[54] J. Li, M. Qiu, Z. Ming, et al., Online optimization for scheduling preemptable tasks on iaas cloud systems, Journal of Parallel and Distributed Computing 72 (5) (2012) 666-677.

Congress on Evolutionary Computation, Vol. 1, IEEE Press, 2003, pp. 498-505.

[60] H. Ishibuchi, T. Yoshida, T. Murata, Balance between genetic search and local search in memetic algorithms for multiobjective permutation flowshop scheduling, IEEE Trans. Evol. Comput. 7 (2003) 204-223.

[61] Y. Mei, X. Li, X. Yao, On investigation of interdependence between sub-problems of the travelling thief problem, Soft Comput. 20 (1) (2016) 157-172.

[62] Y. Richard, R. Liu, Z. Jiang, A memetic algorithm for the open capacitated arc routing problem, Transp. Res. Part E 50 (2013) 53-67.

[63] T. Runarsson, X. Yao, Stochastic ranking for constrained evolutionary optimization, IEEE Trans. Evol. Comput. 4 (3) (2000) 284-294.

[64] X. Chen, L. Feng, Y. Ong, A self-adaptive memeplexes robust search scheme for solving stochastic demands vehicle routing problem, Int. J. Syst. Sci. 43 (7) (2012) 1347-1366. 


\section{Appendix}

In this section, the detailed results obtained by MARD and the other compared algorithms on the test sets are given in Tables 12 - 15 Tables 12 and 13 show the detailed average results, and Tables 14 and 15 show the detailed best results.

In Tables 12 and 13 , the columns $|V|,|E|$ and $\gamma$ stand for the number of vertices, edges and services, and LBV and LB indicates the lower bounds of $n v$ and $t c$, respectively.

In Tables 14 and 15 , the achieved best results are compared against the best-known results (obtained from [31, [32, 33, 34]), which is shown in the column BK. If the best-known result is updated, the new best result is marked with "*”.

Table 12: The average results over 30 independent runs of MARM and MARD and the results of LMA and SS on the pgdb instances. For each instance, the $t$-tests have been conducted between the compared algorithms under the confidence probability of $95 \%$. If an algorithm is significantly better than all the others, then its corresponding entry is marked with "†".

\begin{tabular}{|c|c|c|c|c|c|c|c|c|c|c|c|c|c|}
\hline \multirow[t]{2}{*}{ Name } & \multirow[t]{2}{*}{$|V|$} & \multirow[t]{2}{*}{$|E|$} & \multirow[t]{2}{*}{$\gamma$} & \multirow[t]{2}{*}{ LBV } & \multirow[t]{2}{*}{ LB } & \multicolumn{2}{|c|}{ LMA(IPMA) } & \multicolumn{2}{|c|}{ SS } & \multicolumn{2}{|c|}{$\begin{array}{l}\text { MARM } \\
\text { Average }\end{array}$} & \multicolumn{2}{|c|}{$\begin{array}{l}\text { MARD } \\
\text { Average }\end{array}$} \\
\hline & & & & & & $n v$ & $t c$ & $n v$ & $t c$ & $n v$ & $t c$ & $n v$ & $t c$ \\
\hline 1 & 12 & 44 & 65 & 3 & 771 & 3 & 890 & 3 & 869 & $3.0 \pm 0.0$ & $827.4 \pm 8.6$ & $3.0 \pm 0.0$ & $818.3 \pm 7.3^{\dagger}$ \\
\hline 2 & 12 & 52 & 76 & 3 & 887 & 3 & 1030 & 3 & 997 & $3.0 \pm 0.0$ & $934.6 \pm 12.7$ & $3.0 \pm 0.0$ & $915.2 \pm 9.4^{\dagger}$ \\
\hline 3 & 12 & 44 & 61 & 3 & 673 & 3 & 794 & 3 & 788 & $3.0 \pm 0.0$ & $710.4 \pm 9.9$ & $3.0 \pm 0.0$ & $698.6 \pm 7.3^{\dagger}$ \\
\hline 4 & 11 & 38 & 52 & 2 & 673 & 2 & 858 & 2 & 795 & $2.0 \pm 0.0$ & $762.1 \pm 12.7$ & $2.0 \pm 0.0$ & $744.0 \pm 5.9^{\dagger}$ \\
\hline 5 & 13 & 52 & 75 & 3 & 965 & 3 & 1120 & 3 & 1098 & $3.0 \pm 0.0$ & $1032.4 \pm 14.2$ & $3.0 \pm 0.0$ & $1021.6 \pm 8.6^{\dagger}$ \\
\hline 6 & 12 & 44 & 67 & 3 & 861 & 3 & 1008 & 3 & 951 & $3.0 \pm 0.0$ & $912.2 \pm 7.9$ & $3.0 \pm 0.0$ & $895.6 \pm 6.9^{\dagger}$ \\
\hline 7 & 12 & 44 & 65 & 3 & 801 & 3 & 935 & 3 & 882 & $3.0 \pm 0.0$ & $836.0 \pm 8.1$ & $3.0 \pm 0.0$ & $818.6 \pm 5.2^{\dagger}$ \\
\hline 8 & 27 & 92 & 143 & 5 & 814 & 5 & 1000 & 6 & 1014 & $5.0 \pm 0.0$ & $986.0 \pm 14.7$ & $5.0 \pm 0.2$ & $963.4 \pm 13.9^{\dagger}$ \\
\hline 9 & 27 & 102 & 155 & 5 & 772 & 5 & 934 & 6 & 966 & $5.0 \pm 0.0$ & $917.3 \pm 11.5$ & $5.2 \pm 0.4$ & $892.0 \pm 10.0^{\dagger}$ \\
\hline 10 & 12 & 50 & 65 & 2 & 669 & 2 & 744 & 2 & 761 & $2.0 \pm 0.0$ & $696.3 \pm 9.1$ & $2.0 \pm 0.0$ & $684.4 \pm 4.5^{\dagger}$ \\
\hline 11 & 22 & 90 & 133 & 3 & 1043 & 3 & 1172 & 3 & 1193 & $3.0 \pm 0.0$ & $1113.4 \pm 11.0$ & $3.0 \pm 0.0$ & $1089.7 \pm 7.3^{\dagger}$ \\
\hline 12 & 13 & 46 & 67 & 3 & 1021 & 3 & 1218 & 3 & 1245 & $3.0 \pm 0.0$ & $1149.8 \pm 14.5$ & $3.0 \pm 0.0$ & $1132.8 \pm 12.5^{\dagger}$ \\
\hline 13 & 10 & 56 & 81 & 3 & 1541 & 3 & 1597 & 3 & 1593 & $3.0 \pm 0.0$ & $1564.9 \pm 5.3$ & $3.0 \pm 0.0$ & $1556.5 \pm 3.5^{\dagger}$ \\
\hline 14 & 7 & 42 & 64 & 2 & 278 & 3 & 300 & 3 & 285 & $2.5 \pm 0.5^{\dagger}$ & $291.0 \pm 8.7$ & $3.0 \pm 0.0$ & $282.5 \pm 1.1^{\dagger}$ \\
\hline 15 & 7 & 42 & 64 & 2 & 174 & 2 & 182 & 2 & 180 & $2.0 \pm 0.0$ & $176.2 \pm 1.5$ & $2.0 \pm 0.0$ & $174.0 \pm 0.0^{\dagger}$ \\
\hline 16 & 8 & 56 & 85 & 3 & 352 & 3 & 374 & 3 & 372 & $3.0 \pm 0.0$ & $364.2 \pm 2.1$ & $3.0 \pm 0.0$ & $357.8 \pm 1.3^{\dagger}$ \\
\hline 17 & 8 & 56 & 85 & 2 & 255 & 3 & 267 & 3 & 265 & $2.0 \pm 0.0^{\dagger}$ & $266.1 \pm 2.0$ & $3.0 \pm 0.0$ & $256.8 \pm 0.9^{\dagger}$ \\
\hline 18 & 9 & 72 & 106 & 2 & 484 & 3 & 506 & 3 & 506 & $2.0 \pm 0.0^{\dagger}$ & $494.4 \pm 3.3$ & $3.0 \pm 0.0$ & $485.2 \pm 1.0^{\dagger}$ \\
\hline 19 & 8 & 22 & 30 & 2 & 155 & 2 & 181 & 2 & 173 & $2.0 \pm 0.0$ & $172.7 \pm 0.8$ & $2.0 \pm 0.0$ & $171.5 \pm 0.8^{\dagger}$ \\
\hline 20 & 11 & 44 & 63 & 2 & 339 & 3 & 375 & 3 & 356 & $2.0 \pm 0.0^{\dagger}$ & $357.8 \pm 4.1$ & $2.8 \pm 0.4$ & $348.6 \pm 3.3^{\dagger}$ \\
\hline 21 & 11 & 66 & 101 & 3 & 488 & 3 & 525 & 4 & 509 & $3.0 \pm 0.0$ & $504.9 \pm 3.8$ & $3.2 \pm 0.4$ & $499.7 \pm 4.4^{\dagger}$ \\
\hline 22 & 11 & 88 & 129 & 4 & 578 & 4 & 600 & 5 & 597 & $4.0 \pm 0.0$ & $593.0 \pm 2.7$ & $4.4 \pm 0.5$ & $587.6 \pm 3.0^{\dagger}$ \\
\hline 23 & 11 & 110 & 165 & 5 & 671 & 5 & 691 & 6 & 702 & $5.0 \pm 0.0$ & $691.4 \pm 3.1$ & $5.9 \pm 0.3$ & $681.4 \pm 2.2^{\dagger}$ \\
\hline
\end{tabular}


Table 13: The average results over 30 independent runs of MARM and MARD and the results of SS on the pval instances. For each instance, $t$-tests have been conducted between the compared algorithms under the confidence probability of $95 \%$. If an algorithm is significantly better than all the others, then its corresponding entry is marked with "†".

\begin{tabular}{|c|c|c|c|c|c|c|c|c|c|c|c|}
\hline \multirow[t]{3}{*}{ Name } & \multirow[t]{3}{*}{$|V|$} & \multirow[t]{3}{*}{$|E|$} & \multirow[t]{3}{*}{$\gamma$} & \multirow[t]{3}{*}{ LBV } & \multirow[t]{3}{*}{ LB } & \multirow{2}{*}{\multicolumn{2}{|c|}{ SS }} & \multicolumn{2}{|c|}{ MARM } & \multicolumn{2}{|c|}{ MARD } \\
\hline & & & & & & & & \multicolumn{2}{|c|}{ Average } & \multicolumn{2}{|c|}{ Average } \\
\hline & & & & & & $n v$ & $t c$ & $n v$ & $t c$ & $n v$ & $t c$ \\
\hline $1 \mathrm{a}$ & 24 & 78 & 105 & 2 & 434 & 2 & 544 & $2.0 \pm 0.0$ & $488.8 \pm 6.6$ & $2.0 \pm 0.0$ & $480.4 \pm 5.1^{\dagger}$ \\
\hline $1 \mathrm{~b}$ & 24 & 78 & 105 & 3 & 458 & 3 & 585 & $3.0 \pm 0.0$ & $540.0 \pm 4.7$ & $3.0 \pm 0.0$ & $528.9 \pm 4.2^{\dagger}$ \\
\hline $1 \mathrm{c}$ & 24 & 78 & 105 & 4 & 532 & 5 & 701 & $4.0 \pm 0.0^{\dagger}$ & $678.5 \pm 11.6$ & $4.4 \pm 0.5$ & $658.3 \pm 11.0^{\dagger}$ \\
\hline $2 \mathrm{a}$ & 24 & 68 & 94 & 2 & 606 & 2 & 782 & $2.0 \pm 0.0$ & $706.0 \pm 5.0$ & $2.0 \pm 0.0$ & $700.4 \pm 2.0^{\dagger}$ \\
\hline $2 \mathrm{~b}$ & 24 & 68 & 94 & 2 & 695 & 3 & 868 & $2.0 \pm 0.0$ & $788.8 \pm 6.7$ & $2.0 \pm 0.0$ & $781.0 \pm 4.1^{\dagger}$ \\
\hline $2 \mathrm{c}$ & 24 & 68 & 94 & 4 & 1012 & 5 & 1259 & $4.0 \pm 0.0$ & $1183.3 \pm 16.1$ & $4.0 \pm 0.0$ & $1174.1 \pm 13.3^{\dagger}$ \\
\hline $3 a$ & 24 & 70 & 96 & 2 & 192 & 2 & 249 & $2.0 \pm 0.0$ & $225.9 \pm 2.3$ & $2.0 \pm 0.0$ & $223.7 \pm 2.3$ \\
\hline $3 b$ & 24 & 70 & 96 & 2 & 212 & 3 & 278 & $2.0 \pm 0.0^{\dagger}$ & $263.8 \pm 5.0$ & $2.4 \pm 0.5$ & $261.3 \pm 6.9$ \\
\hline $3 \mathrm{c}$ & 24 & 70 & 96 & 4 & 289 & 4 & 358 & $4.0 \pm 0.0$ & $347.1 \pm 4.5$ & $4.0 \pm 0.0$ & $343.4 \pm 4.7^{\dagger}$ \\
\hline $4 \mathrm{a}$ & 41 & 138 & 205 & 2 & 1089 & 3 & 1330 & $2.0 \pm 0.0^{\dagger}$ & $1262.4 \pm 18.6$ & $2.8 \pm 0.4$ & $1204.9 \pm 15.0^{\dagger}$ \\
\hline $4 \mathrm{~b}$ & 41 & 138 & 205 & 3 & 1113 & 4 & 1471 & $3.0 \pm 0.0$ & $1314.7 \pm 14.0$ & $3.0 \pm 0.0$ & $1289.2 \pm 15.1^{\dagger}$ \\
\hline $4 \mathrm{c}$ & 41 & 138 & 205 & 4 & 1205 & 4 & 1583 & $4.0 \pm 0.0$ & $1438.7 \pm 13.6$ & $4.0 \pm 0.0$ & $1410.2 \pm 13.9^{\dagger}$ \\
\hline $4 \mathrm{~d}$ & 41 & 138 & 205 & 6 & 1502 & 8 & 1988 & $6.1 \pm 0.3^{\dagger}$ & $1905.6 \pm 31.0$ & $7.0 \pm 0.2$ & $1831.2 \pm 17.3^{\dagger}$ \\
\hline $5 \mathrm{a}$ & 34 & 130 & 194 & 2 & 1184 & 3 & 1454 & $2.0 \pm 0.0^{\dagger}$ & $1353.3 \pm 18.8$ & $2.9 \pm 0.2$ & $1296.2 \pm 15.3^{\dagger}$ \\
\hline $5 \mathrm{~b}$ & 34 & 130 & 194 & 3 & 1241 & 4 & 1528 & $3.0 \pm 0.0$ & $1417.6 \pm 15.6$ & $3.0 \pm 0.0$ & $1401.4 \pm 13.4^{\dagger}$ \\
\hline $5 \mathrm{c}$ & 34 & 130 & 194 & 4 & 1348 & 4 & 1655 & $4.0 \pm 0.0$ & $1541.9 \pm 9.6$ & $4.0 \pm 0.0$ & $1516.5 \pm 11.9^{\dagger}$ \\
\hline $5 \mathrm{~d}$ & 34 & 130 & 194 & 6 & 1638 & 7 & 2097 & $6.0 \pm 0.0^{\dagger}$ & $2033.3 \pm 21.9$ & $6.6 \pm 0.5$ & $1980.5 \pm 37.5^{\dagger}$ \\
\hline $6 \mathrm{a}$ & 31 & 100 & 150 & 2 & 658 & 3 & 754 & $2.0 \pm 0.0^{\dagger}$ & $741.4 \pm 8.5$ & $2.4 \pm 0.5$ & $731.6 \pm 10.6^{\dagger}$ \\
\hline $6 \mathrm{~b}$ & 31 & 100 & 150 & 3 & 684 & 4 & 863 & $3.0 \pm 0.0$ & $786.4 \pm 7.5$ & $3.0 \pm 0.0$ & $769.4 \pm 8.4^{\dagger}$ \\
\hline $6 \mathrm{c}$ & 31 & 100 & 150 & 7 & 921 & 8 & 1183 & $7.0 \pm 0.0$ & $1139.0 \pm 11.3$ & $7.0 \pm 0.0$ & $1119.7 \pm 10.7^{\dagger}$ \\
\hline $7 \mathrm{a}$ & 40 & 132 & 201 & 2 & 833 & 3 & 1040 & $2.0 \pm 0.2^{\dagger}$ & $997.7 \pm 24.4$ & $3.0 \pm 0.0$ & $924.1 \pm 7.5^{\dagger}$ \\
\hline $7 \mathrm{~b}$ & 40 & 132 & 201 & 3 & 835 & 4 & 1046 & $3.0 \pm 0.0^{\dagger}$ & $978.5 \pm 11.2$ & $3.4 \pm 0.5$ & $955.4 \pm 18.3^{\dagger}$ \\
\hline $7 \mathrm{c}$ & 40 & 132 & 201 & 7 & 949 & 8 & 1283 & $7.0 \pm 0.0$ & $1190.8 \pm 14.5$ & $7.0 \pm 0.0$ & $1153.2 \pm 10.6^{\dagger}$ \\
\hline $8 \mathrm{a}$ & 30 & 126 & 194 & 2 & 1150 & 3 & 1343 & $2.7 \pm 0.5^{\dagger}$ & $1282.3 \pm 47.1$ & $3.0 \pm 0.0$ & $1226.6 \pm 8.8^{\dagger}$ \\
\hline $8 \mathrm{~b}$ & 30 & 126 & 194 & 3 & 1197 & 4 & 1453 & $3.0 \pm 0.0$ & $1330.4 \pm 14.8$ & $3.0 \pm 0.0$ & $1295.6 \pm 12.8^{\dagger}$ \\
\hline $8 \mathrm{c}$ & 30 & 126 & 194 & 7 & 1567 & 7 & 1970 & $7.0 \pm 0.0$ & $1886.0 \pm 14.6$ & $7.0 \pm 0.0$ & $1852.2 \pm 16.8^{\dagger}$ \\
\hline $9 \mathrm{a}$ & 50 & 184 & 274 & 2 & 867 & 3 & 1083 & $2.0 \pm 0.2^{\dagger}$ & $990.0 \pm 13.4$ & $3.0 \pm 0.2$ & $931.4 \pm 8.8^{\dagger}$ \\
\hline $9 b$ & 50 & 184 & 274 & 3 & 891 & 4 & 1087 & $3.0 \pm 0.0$ & $1014.0 \pm 9.6$ & $3.0 \pm 0.0$ & $970.5 \pm 10.4^{\dagger}$ \\
\hline $9 \mathrm{c}$ & 50 & 184 & 274 & 4 & 913 & 4 & 1195 & $4.0 \pm 0.0$ & $1050.9 \pm 12.3$ & $4.0 \pm 0.0$ & $1007.0 \pm 12.3^{\dagger}$ \\
\hline $9 \mathrm{~d}$ & 50 & 184 & 274 & 7 & 1064 & 8 & 1418 & $7.0 \pm 0.0^{\dagger}$ & $1367.2 \pm 23.4$ & $7.9 \pm 0.3$ & $1294.6 \pm 13.6^{\dagger}$ \\
\hline $10 \mathrm{a}$ & 50 & 194 & 300 & 2 & 1247 & 3 & 1478 & $2.3 \pm 0.4^{\dagger}$ & $1401.8 \pm 31.6$ & $3.0 \pm 0.0$ & $1347.8 \pm 9.8^{\dagger}$ \\
\hline $10 \mathrm{~b}$ & 50 & 194 & 300 & 3 & 1259 & 4 & 1580 & $3.0 \pm 0.0$ & $1415.9 \pm 11.2$ & $3.0 \pm 0.0$ & $1387.5 \pm 10.4^{\dagger}$ \\
\hline $10 \mathrm{c}$ & 50 & 194 & 300 & 4 & 1301 & 4 & 1613 & $4.0 \pm 0.0$ & $1477.1 \pm 11.0$ & $4.0 \pm 0.0$ & $1442.8 \pm 11.2^{\dagger}$ \\
\hline $10 \mathrm{~d}$ & 50 & 194 & 300 & 7 & 1551 & 9 & 1964 & $7.0 \pm 0.0^{\dagger}$ & $1879.8 \pm 21.0$ & $7.9 \pm 0.3$ & $1814.4 \pm 14.2^{\dagger}$ \\
\hline
\end{tabular}


Table 14: The best results of the compared algorithms on 23 pgdb instances. For MARM and MARD, "Best" stands for the best results obtained by 30 runs, while for LMA and SS, the results were obtained by one run using sophisticated parameter settings. For each instance, the best results among all the compared results are marked in bold, and the new best results obtained by MARD are marked with "*”.

\begin{tabular}{|c|c|c|c|c|c|c|c|c|c|c|c|c|}
\hline \multirow[t]{3}{*}{ Name } & \multirow[t]{3}{*}{ LBV } & \multirow[t]{3}{*}{ LB } & \multirow{2}{*}{\multicolumn{2}{|c|}{ LMA(IPMA) }} & \multirow{2}{*}{\multicolumn{2}{|c|}{ SS }} & \multirow{2}{*}{\multicolumn{2}{|c|}{$\frac{\text { MARM }}{\text { Best }}$}} & \multirow{2}{*}{\multicolumn{2}{|c|}{$\frac{\text { MARD }}{\text { Best }}$}} & \multirow{2}{*}{\multicolumn{2}{|c|}{ BK }} \\
\hline & & & & & & & & & & & & \\
\hline & & & $n v$ & $t c$ & $n v$ & $t c$ & $n v$ & $t c$ & $n v$ & $t c$ & $n v$ & $t c$ \\
\hline 1 & 3 & 771 & 3 & 890 & 3 & 869 & 3 & 810 & 3 & $799 *$ & 3 & 810 \\
\hline 2 & 3 & 887 & 3 & 1030 & 3 & 997 & 3 & 917 & 3 & $899 *$ & 3 & 917 \\
\hline 3 & 3 & 673 & 3 & 794 & 3 & 788 & 3 & 691 & 3 & $687^{*}$ & 3 & 691 \\
\hline 4 & 2 & 673 & 2 & 858 & 2 & 795 & 2 & 740 & 2 & $729 *$ & 2 & 740 \\
\hline 5 & 3 & 965 & 3 & 1120 & 3 & 1098 & 3 & 1004 & 3 & $1003^{*}$ & 3 & 1004 \\
\hline 6 & 3 & 861 & 3 & 1008 & 3 & 951 & 3 & 900 & 3 & $881^{*}$ & 3 & 900 \\
\hline 7 & 3 & 801 & 3 & 935 & 3 & 882 & 3 & 819 & 3 & $813^{*}$ & 3 & 819 \\
\hline 8 & 5 & 814 & 5 & 1000 & 6 & 1014 & 5 & 953 & 5 & $928^{*}$ & 5 & 953 \\
\hline 9 & 5 & 772 & 5 & 934 & 6 & 966 & 5 & 892 & 5 & $864^{*}$ & 5 & 892 \\
\hline 10 & 2 & 669 & 2 & 744 & 2 & 761 & 2 & 677 & 2 & $675^{*}$ & 2 & 677 \\
\hline 11 & 3 & 1043 & 3 & 1172 & 3 & 1193 & 3 & 1089 & 3 & $1075^{*}$ & 3 & 1089 \\
\hline 12 & 3 & 1021 & 3 & 1218 & 3 & 1245 & 3 & 1118 & 3 & $1104^{*}$ & 3 & 1118 \\
\hline 13 & 3 & 1541 & 3 & 1597 & 3 & 1593 & 3 & 1555 & 3 & $1551^{*}$ & 3 & 1555 \\
\hline 14 & 2 & 278 & 3 & 300 & 3 & 285 & 2 & 290 & 3 & $280^{*}$ & 2 & 290 \\
\hline 15 & 2 & 174 & 2 & 182 & 2 & 180 & 2 & 174 & 2 & 174 & 2 & 174 \\
\hline 16 & 3 & 352 & 3 & 374 & 3 & 372 & 3 & 360 & 3 & $356^{*}$ & 3 & 360 \\
\hline 17 & 2 & 255 & 3 & 267 & 3 & 265 & 2 & 261 & 3 & 255 & 2 & 261 \\
\hline 18 & 2 & 484 & 3 & 506 & 3 & 506 & 2 & 487 & 3 & 484 & 2 & 487 \\
\hline 19 & 2 & 155 & 2 & 181 & 2 & 173 & 2 & 171 & 2 & 171 & 2 & 171 \\
\hline 20 & 2 & 339 & 3 & 375 & 3 & 356 & 2 & 348 & 3 & $345^{*}$ & 2 & 348 \\
\hline 21 & 3 & 488 & 3 & 525 & 4 & 509 & 3 & 498 & 3 & $496 *$ & 3 & 498 \\
\hline 22 & 4 & 578 & 4 & 600 & 5 & 597 & 4 & 589 & 4 & $583^{*}$ & 4 & 589 \\
\hline 23 & 5 & 671 & 5 & 691 & 6 & 702 & 5 & 686 & 5 & $675^{*}$ & 5 & 686 \\
\hline
\end{tabular}


Table 15: The best results of the compared algorithms on 34 pval instances. For MARM and MARD, "Best" stands for the best results obtained by 30 runs, while for LMA and SS, the results were obtained by one run using sophisticated parameter settings. For each instance, the best results among all the compared results are marked in bold, and the new best results obtained by MARD are marked with "*”.

\begin{tabular}{|c|c|c|c|c|c|c|c|c|c|c|}
\hline \multirow[t]{3}{*}{ Name } & \multirow[t]{3}{*}{ LBV } & \multirow[t]{3}{*}{ LB } & \multirow{2}{*}{\multicolumn{2}{|c|}{ SS }} & \multirow{2}{*}{\multicolumn{2}{|c|}{$\frac{\text { MARM }}{\text { Best }}$}} & \multirow{2}{*}{\multicolumn{2}{|c|}{$\frac{\text { MARD }}{\text { Best }}$}} & \multirow{2}{*}{\multicolumn{2}{|c|}{ BK }} \\
\hline & & & & & & & & & & \\
\hline & & & $n v$ & $t c$ & $n v$ & $t c$ & $n v$ & $t c$ & $n v$ & $t c$ \\
\hline $1 \mathrm{a}$ & 2 & 434 & 2 & 544 & 2 & 470 & 2 & $466^{*}$ & 2 & 470 \\
\hline $1 b$ & 3 & 458 & 3 & 585 & 3 & 530 & 3 & $518^{*}$ & 3 & 530 \\
\hline $1 \mathrm{c}$ & 4 & 532 & 5 & 701 & 4 & 653 & 4 & $646^{*}$ & 4 & 653 \\
\hline $2 \mathrm{a}$ & 2 & 606 & 2 & 782 & 2 & 697 & 2 & 697 & 2 & 697 \\
\hline $2 b$ & 2 & 695 & 3 & 868 & 2 & 775 & 2 & $\mathbf{7 7 3}^{*}$ & 2 & 775 \\
\hline $2 \mathrm{c}$ & 4 & 1012 & 5 & 1259 & 4 & 1149 & 4 & $1140 *$ & 4 & 1149 \\
\hline $3 a$ & 2 & 192 & 2 & 249 & 2 & 222 & 2 & $219^{*}$ & 2 & 222 \\
\hline $3 b$ & 2 & 212 & 3 & 278 & 2 & 255 & 2 & $249^{*}$ & 2 & 255 \\
\hline $3 c$ & 4 & 289 & 4 & 358 & 4 & 336 & 4 & $333^{*}$ & 4 & 336 \\
\hline $4 \mathrm{a}$ & 2 & 1089 & 3 & 1330 & 2 & 1228 & 2 & $1201^{*}$ & 2 & 1228 \\
\hline $4 b$ & 3 & 1113 & 4 & 1471 & 3 & 1288 & 3 & $1265^{*}$ & 3 & 1288 \\
\hline $4 \mathrm{c}$ & 4 & 1205 & 4 & 1583 & 4 & 1409 & 4 & $1382^{*}$ & 4 & 1409 \\
\hline $4 d$ & 6 & 1502 & 8 & 1988 & 6 & 1858 & 6 & 1858 & 6 & 1858 \\
\hline $5 \mathrm{a}$ & 2 & 1184 & 3 & 1454 & 2 & 1315 & 2 & $1300^{*}$ & 2 & 1315 \\
\hline $5 b$ & 3 & 1241 & 4 & 1528 & 3 & 1384 & 3 & $1378^{*}$ & 3 & 1384 \\
\hline $5 c$ & 4 & 1348 & 4 & 1655 & 4 & 1522 & 4 & $1498^{*}$ & 4 & 1522 \\
\hline $5 \mathrm{~d}$ & 6 & 1638 & 7 & 2097 & 6 & 1991 & 6 & $1950^{*}$ & 6 & 1991 \\
\hline $6 \mathrm{a}$ & 2 & 658 & 3 & 754 & 2 & 722 & 3 & $719^{*}$ & 2 & 722 \\
\hline $6 \mathrm{~b}$ & 3 & 684 & 4 & 863 & 3 & 774 & 3 & $754^{*}$ & 3 & 774 \\
\hline $6 \mathrm{c}$ & 7 & 921 & 8 & 1183 & 7 & 1117 & 7 & $1098^{*}$ & 7 & 1117 \\
\hline $7 \mathrm{a}$ & 2 & 833 & 3 & 1040 & 2 & 966 & 3 & $912^{*}$ & 2 & 966 \\
\hline $7 \mathrm{~b}$ & 3 & 835 & 4 & 1046 & 3 & 960 & 3 & $947^{*}$ & 3 & 960 \\
\hline $7 \mathrm{c}$ & 7 & 949 & 8 & 1283 & 7 & 1165 & 7 & $1137^{*}$ & 7 & 1165 \\
\hline $8 \mathrm{a}$ & 2 & 1150 & 3 & 1343 & 2 & 1292 & 3 & $1203^{*}$ & 2 & 1292 \\
\hline $8 b$ & 3 & 1197 & 4 & 1453 & 3 & 1301 & 3 & $1267^{*}$ & 3 & 1301 \\
\hline $8 c$ & 7 & 1567 & 7 & 1970 & 7 & 1853 & 7 & $1816^{*}$ & 7 & 1853 \\
\hline $9 \mathrm{a}$ & 2 & 867 & 3 & 1083 & 2 & 966 & 2 & 966 & 2 & 966 \\
\hline $9 \mathrm{~b}$ & 3 & 891 & 4 & 1087 & 3 & 990 & 3 & $951^{*}$ & 3 & 990 \\
\hline $9 c$ & 4 & 913 & 4 & 1195 & 4 & 1031 & 4 & 982* & 4 & 1031 \\
\hline $9 \mathrm{~d}$ & 7 & 1064 & 8 & 1418 & 7 & 1324 & 7 & $1278^{*}$ & 7 & 1324 \\
\hline $10 \mathrm{a}$ & 2 & 1247 & 3 & 1478 & 2 & 1385 & 3 & $1330 *$ & 2 & 1385 \\
\hline $10 \mathrm{~b}$ & 3 & 1259 & 4 & 1580 & 3 & 1395 & 3 & $1367^{*}$ & 3 & 1395 \\
\hline $10 \mathrm{c}$ & 4 & 1301 & 4 & 1613 & 4 & 1461 & 4 & $1411^{*}$ & 4 & 1461 \\
\hline $10 \mathrm{~d}$ & 7 & 1551 & 9 & 1964 & 7 & 1837 & 7 & $1817^{*}$ & 7 & 1837 \\
\hline
\end{tabular}

